\title{
1 Functions of anionic lipids in plants
}

2

3 Lise C. Noack and Yvon Jaillais

4

5 Laboratoire Reproduction et Développement des Plantes, Univ Lyon, ENS de Lyon, UCB

6 Lyon 1, CNRS, INRA, F-69342 Lyon, France

7 Corresponding author: Yvon Jaillais (yvon.jaillais@ens-lyon.fr)

8 Lise C. Noack (lise.noack@ens-lyon.fr) : ORCID\# 0000-0001-5093-6374

9 Yvon Jaillais (yvon.jaillais@ens-lyon.fr) : ORCID\# 0000-0003-4923-883X

10

11 
Abstract: (150 words max)

13 Anionic phospholipids, which include phosphatidic acid, phosphatidylserine, and 14 phosphoinositides, represent a low percentage of membrane lipids. They are able to modulate 15 the physical properties of membranes, such as their surface charges, curvature or clustering of 16 proteins. Moreover, by mediating interactions with numerous membrane-associated proteins, 17 they are key components in the establishment of organelle identity and dynamics. Finally, 18 anionic lipids also act as signalling molecules, as they are rapidly produced or interconverted 19 by a set of dedicated enzymes. As such, anionic lipids are major regulators of many 20 fundamental cellular processes, including cell signalling, cell division, membrane trafficking, 21 cell growth or gene expression. In this review, we describe the functions of anionic lipids with 22 a cellular perspective. Using the localization of each anionic lipids and their related metabolic 23 enzymes as starting points, we summarize their roles within the different compartments of the 24 endomembrane system and address their associated developmental and physiological 25 consequences.

27 Keywords: (6 max)

28 membrane contact sites, endocytosis, exocytosis, autophagy, nanodomains, plant-microbe 29 interphase 


\section{INTRODUCTION}

34 Biological membranes delimit cellular compartments, as well as the inner and outer of the cell. Each compartment has its proper chemical environment allowing numerous and specialized chemical reactions in the same cell. To enable the proper localization of proteins, each membrane has its own biochemical and biophysical identity. Membranes are composed of lipids forming a bilayer and transmembrane or membrane-associated proteins (63). Glycerophospholipids associated with sphingolipids and sterols are the main lipid components within the eukaryotic endomembrane system. Among the glycerophospholipids some present negative charges and, as such, are anionic phospholipids. Anionic phospholipids include phosphatidic acid (PA), phosphatidylserine (PS), phosphatidylinositol and phosphatidylinositol phosphates (PIPs also referred to as phosphoinositides), the phosphorylated forms of phosphatidylinositol (Figure 1.a). Phosphatidylinositol display an inositol ring that can be phosphorylated in three different positions - 3, 4 and 5 - creating a total of seven possible phosphoinositide species: 3 phosphoinositide monophosphates (PI3P, PI4P, PI5P), 3 phosphoinositide biphosphates $\left(\mathrm{PI}(3,4) \mathrm{P}_{2}, \mathrm{PI}(3,5) \mathrm{P}_{2}, \mathrm{PI}(4,5) \mathrm{P}_{2}\right)$ and one phosphoinositide triphosphate $\left(\mathrm{PI}(3,4,5) \mathrm{P}_{3}\right)$.

These lipids are present in low to very low abundance in eukaryotic membranes. In Arabidopsis leaf, phosphatidylinositol represents about $7 \%$ of total phospholipids, PA $\sim 2 \%$ and PS $\sim 1 \%$. In addition, all phosphoinositides combined account for less than $1 \%$ of the total phospholipid content $(72,135)$. However, anionic lipids are key components of cell membranes. They are

54 involved in the regulation of nearly all membrane-associated events including cell signalling,

55 trafficking, cell division, and cell growth. Consequently, over-expression lines or loss-of-function 56 mutants for lipid enzymes that perturb anionic lipid homeostasis lead to severe and pleiotropic 57 phenotypes at the plant level, including defects in development, immunity, reproduction and stress adaptation $(24,94)$. 
Anionic phospholipids are detected in most endomembrane compartments: endoplasmic reticulum (ER), Golgi, trans-Golgi network/early endosomes (TGN/EE), plasma membrane (PM), Late endosomes/multi-vesicular bodies (LE/MVB), vacuoles and autophagosomes. In this review, we first describe the key physicochemical properties of anionic lipids that mediate lipid-protein interactions and regulate protein dynamics in cell membranes. Then, we discuss the functions of anionic lipids with a cellular perspective, using as a starting point the localization of each anionic lipids and their related metabolic enzymes. We summarize the roles of anionic lipids within the different compartments of the endomembrane system and address their associated developmental and physiological consequences. It should be noted that anionic lipids also accumulate in mitochondria and plastids where they play a role in division, lipid synthesis and photosynthesis. Anionic lipids, such as PA, PI4P and $\mathrm{PI}(4,5) \mathrm{P}_{2}$ are also found in the nucleoplasm of the nucleus where they may regulate transcription factors, chromatin status and epigenetic landmarks (125) but this will not be further discussed, as we decided to focus this review on the endomembrane system.

Cell compartments are enriched in different anionic lipids, which give specific properties to their membrane and thereby contribute to their identity. Lipids binding domains (LBDs) can mediate the interaction with the membrane $(63,126)$ (Figure 2.d). They allow the targeting of proteins to membranes by stereospecific interactions with a given phospholipid species. Those domains are, for example, Pleckstrin Homology (PH) domains, Fab1/YOTB/Vac1/EEA1 (FYVE) domains, Phox (PX) domains and C2 domains. The characterization of these domains and their specificity allowed the engineering of a set of anionic phospholipid biosensors by fusion of these domains with a fluorescent protein (92). These so called genetically encoded biosensors enable 
phospholipids in vivo $(112,127-129)$. However, in some cases, lipid-binding domains are not sufficient to target the protein to its proper compartment. Indeed, some proteins require the present of both, an anionic lipid and another protein (or lipid) to localize to the right compartment, in a mechanism called "coincidence binding" (Figure 2.d). For instance, the ubiquitin ligase PLANT-U-BOX13 (PUB13), which regulates the trafficking of the PM receptor FLAGELIN-INSENSITIVE 2 (FLS2), is recruited to the TGN via the concomitant interaction with RabA4b and PI4P (2). Furthermore, LBDs may also recognize additional membrane feature such as curvature or electrostatics. As such, results obtained with lipid biosensors should always been taken with care as their localization may not only reflect the accumulation of a single lipid species. This is exemplified by the fact that biosensors designed to bind to the same lipid species do not always have the exact same localization $(112,113)$. If possible, the potential accumulation of a lipid at a given membrane should be also supported by biochemical evidences and/or by the localization of their metabolic enzymes.

The combined study of the localization of anionic lipid generating enzymes and genetically encoded lipid sensors suggest that in Arabidopsis epidermal root cells, the PM accumulates PI4P, $\mathrm{PI}(4,5) \mathrm{P}_{2}, \mathrm{PA}$ and PS (Figure 3) $(93,112,113,127,128)$. TGN/EE are enriched in PI4P, PS and to a lesser extent PI3P $(93,113,114,128)$. LE/MVBs accumulate both PI3P and PI(3,5) $\mathrm{P}_{2}$, although both lipids localize in distinct compartments $(37,38,112,129)$. Finally, the tonoplast and autophagosomes carry PI3P $(112,129)$. However, this subcellular accumulation pattern may vary according to the cell type or the stress status of the cell (Figure 3$)(34,41,44,48,57$ in revision, $61,110,115)$. For example, $\mathrm{PI}(3,5) \mathrm{P}_{2}$ is found in LE/MVBs in most cell type but accumulates at the $\mathrm{PM}$ in elongating root hairs (34) (Figure 3). PI(4,5) $\mathrm{P}_{2}$ and $\mathrm{PA}$ are constitutively produced at the PM at low or moderate levels, respectively, in resting condignition but upon stimulation, such as salt stress, they can be massively produced, which triggers specific stress responses $(82,121,127)$. Alternatively, $\mathrm{PI}(4,5) \mathrm{P}_{2}$ production can be inhibited by external stimuli such as pathogen-associated molecular patterns (80). 


\subsubsection{PA production and stress responses}

118 PA is structurally the simplest phospholipid. As such, it is a key intermediate for other 119 phospholipid biosynthesis $(94,121)$. Multiple pathways involving different enzymes at various 120 locations can lead to PA synthesis and are summarized in Figure 1.b. The rapid accumulation of 121 PA is induced by numerous abiotic stresses including salt, cold, heat, or osmotic stress, but also by biotic stresses in case of pathogen infection (121). Moreover, PA synthesis is regulated in physiological and developmental context including hormonal responses such as SA, ABA, and auxin (121). In each case, PA accumulation leads to different adapted cellular responses raising the question: how can PA integrate different inputs to lead to specific outputs relevant to the physiological situation? One of the main hypothesis is that PA accumulation never occurs alone but rather that PA is one second messenger among others that together lead to concerted cellular responses (94). To know more about this topic, the different hypotheses to answer this question are discussed by Pokotylo et al. in a recent review.

\subsubsection{PIPs and rapid inter-conversion}

132 The phosphorylation of the inositol ring of phosphatidylinositol is mediated by PI-kinases that 133 are specific of the phosphate position (Figure 1.d). In plants, the genome does not encode for 134 type-I and type-II PI3-kinases (PI3Ks), able to phosphorylate PI4P or PI(4,5) $\mathrm{P}_{2}(84)$. As a 135 consequence, $\mathrm{PI}(3,4) \mathrm{P}_{2}$ and $\mathrm{PI}(3,4,5) \mathrm{P}_{3}$ likely do not exist and have not been detected in plants.

136 The dephosphorylation of PIPs is mediated by PI-phosphatases that belong in plants to three 137 main families, the SUPPRESSOR OF ACTIN (SAC) domain-containing proteins (SAC1 to SAC9), 138 the 5PTase family (5PTase1 to 15) and the PHOPHATASE AND TENSIN Homolog (PTEN) family 139 (PTEN1 to 3) (24) (Figure 1.d). PI-kinases and phosphatases are highly processive enzymes in 
vivo and thereby PIPs can be quickly interconverted, cycling between two states or creating

141 cascade regulations (86). Because anionic lipid pools can be enzymatically modified within

142 second, they are able to respond to various stimuli and to act in fast cellular events such as

143 membrane trafficking.

145 In vitro experiments testing PI-kinases activity often require the use of detergents, which 146 destroy liposomal structures and free lipid substrates in order to increase kinase activity (52). 147 These results suggest that PI-kinases have difficulties to recognize their substrate embedded 148 into the membrane and might get "help" in planta. The main candidates to fulfil this function are 149 the SEC14 lipid transfer proteins. The yeast Sec14 protein is able to transfer 150 phosphatidylcholine and phosphatidylinositol from distinct membranes in vitro, possibly by151 passing the classical vesicular transport of lipids $(52,103)$. However, the current model, based 152 on crystallographic data, advocates the idea that Sec14 dislodges the phosphatidylinositol head 153 from the TGN membrane making it accessible for PI4-kinases (Figure 4.a). Based on sequence 154 homology, Arabidopsis contains 32 Sec Fourteen Homologs (AtSFHs), each containing a highly 155 conserved sec14 domain. The sec14 domain of several AtSFHs displays the same in vitro activity 156 than the yeast sec14 and can rescue the sec14 yeast mutant $(40,130)$. So far, the best157 characterized AtSFH protein is AtSFH1. AtSFH1 belongs to a subclass of 13 members that 158 contains a Nlj16 domain in C-terminal. This domain has two functions. It contains a polybasic 159 patch that localizes at the $\mathrm{PM}$ in a $\mathrm{PI}(4,5) \mathrm{P}_{2}$-dependent manner and a coiled-coiled domain that 160 mediates homo-oligomerization (25). The model built with the combination of yeast and 161 Arabidopsis data is that AtSFH1 is targeted at the root hair tip PM by $\mathrm{PI}(4,5) \mathrm{P}_{2}$ where it 162 stimulates the activity of PI-kinases (PI4K or PIP5K), thereby enhancing the production of PIPs 163 (directly $\mathrm{PI}(4,5) \mathrm{P}_{2}$ or PI4P that will then be converted into $\left.\mathrm{PI}(4,5) \mathrm{P}_{2}\right)$. PI(4,5) $\mathrm{P}_{2}$ recruit new 164 AtSFH1 which oligomerizes and further creates PI(4,5) $\mathrm{P}_{2}$ clusters at the root hair tip PM (Figure $1653)$. 


\subsection{PS synthesis and trafficking}

169 Contrary to PA and PIPs that can be synthesized by several enzymes, a single enzyme produces 170 PS in Arabidopsis: the Phosphatidylserine Synthase 1 (PSS1) (91, 93, 140) (Figure 1.b). PSS1 is 171 an ER transmembrane protein which synthetizes PS in the luminal leaflet of this compartment 172 (140). Therefore, by contrast to PIPs, PS is not locally produced directly at the membranes 173 where it acts. This implies that PS must be transported from the luminal leaflet of the ER to the 174 cytosolic leaflet of the endomembrane system. Two non-exclusive mechanisms may explain the localization of PS.

\subsubsection{Flippases and vesicular trafficking}

178 As PS faces the lumen in the ER but the cytosol once at the PM or endosomes, it must switch 179 membrane leaflet at some point. Flippases are proteins able to flip phospholipids from the 180 luminal membrane leaflet to the cytosolic membrane leaflet (85) (Figure 4.b). The Arabidopsis 181 genome encodes for 12 aminophospholipid flippases (ALAs) and at least ALA1, ALA2, ALA3 and 182 ALA10 are able to flip PS in yeast $(27,70,71,85,97)$. ALA proteins localize in the ER (ALA1, 2 183 and 3) but also all along the endocytic pathway (ALA1 at the PM, ALA2 in LE/MVBs, ALA3 in the 184 Golgi and TGN/EE) (9, 70, 71, 74, 96). Their localization, and function, depends on ALA 185 interacting $\beta$-subunits, ALIS. These localization suggest that PS could be directly flipped in the 186 ER and then be transported through vesicular trafficking to the PM and endosomes. Another hypothesis would be that PS is first transported by vesicular trafficking on the luminal side of organelle and then flipped in specific compartments, such as TGN/EE or PM. In yeast, the activity

189 of the PS flippase Drs2p is promoted by PI4P $(4,123)$. As PI4P is present in the same 190 compartments than PS along the endocytic pathway in plants, this could be an argument in 191 favour of the later hypothesis. In any case, if the flippase activity occurs at the ER membrane, PS 192 is then actively exported out of this compartment, since the ER cytosolic leaflet does not 193 accumulate PS $(93,113)$. 
196 Another hypothesis suggests that PS bypasses classical vesicular trafficking and rather 197 directly translocates from the ER to the PM, at membrane contact sites (MCSs). MCSs are 198 static microdomains where membranes from two different organelles are bring together (at a distance lower than 30nm) and stabilize by tethering proteins (107). In animal and yeast, MCSs are major sites of lipid flow and are particularly important to control the localization of nearly all anionic lipids (138). Lipid transfer proteins present at MCS are able to transport 202 lipids between two membranes extracting the lipid from the donor membrane, transferring it 203 through the cytosol and inserting it in the acceptor membrane. Notably, proteins from the oxysterol-binding related protein (ORP) family localize at MCS via interactions with suppressor of choline sensitivity Scs2p (VAPS in animal cells) in the ER and PIPs at the PM 206 (3, 138). Several ORPs transfer PS (among other lipids) from the ER to the PM and then 207 counter transport PI4P from the PM back to the ER (Figure 4.c). The PI4P phosphatase 208 SAC1, an ER resident protein, hydrolyses the incoming PI4P, thereby maintaining the PI4P 209 gradient between the PM and ER membrane and fuelling PS export from the ER (3). Note that 210 PS transfer at MCS has not been described so far in plants but that ORP proteins are 211 conserved in plant genomes.

\section{ANIONIC LIPIDS AS DETERMINANT OF MEMBRANE PHYSICOCHEMICAL} PROPERTIES

\subsection{Surface charges}

217 As they carry negative charges, anionic phospholipids generate an electrostatic field on the cytosolic leaflet of membranes (76) (Figure 1.a and 2.b). The strength of this electrostatic 
219 field depends upon the relative concentration in anionic phospholipids and their respective 220 negative charges, which vary from -1 for PS to -5 for PI(4,5) $\mathrm{P}_{2}$. In plants, TGN/EEs are less 221 electronegative than the PM but more electrostatics than MVB/LEs, whereas ER-derived 222 compartments (ER, nuclear membrane, peroxisomes) are neutral $(93,113)$. Consequently, an 223 electrostatic gradient is formed from the PM to the tonoplast, which follows the endocytic 224 route and specifies an "electrostatic membrane territory" within the endomembrane system 225 (93). The extensive use of biosensors locating anionic lipids and assessing membrane surface charge in vivo shows that the high electronegativity of the plant PM relies concomitantly on

227 PI4P, PS and PA, with a major role of PI4P, whereas the surface charge of TGN/EE depends 228 of both PI4P and PS $(93,113)$.

229 The surface charges of membrane compartments are a key determinant to control the 230 localization of many membrane-associated proteins. For example, proteins which contain 231 polybasic patches interact with the negative charges of the anionic phospholipids of the 232 membrane by non-specific electrostatic interactions (Figure 2.d). Very often, such motifs also 233 contain hydrophobic residues or are found adjacent to hydrophobic lipid anchors. For 234 example, the plant AGC kinases PINOID and D6PK, which are well-characterized regulators 235 of auxin efflux transporters from the PIN family, localize at the PM through this mechanism $236(5,61,113)$. Another prominent example of plant proteins, which rely, at least in part, on 237 electrostatic interactions with anionic lipids for localization is the small GTPases from the 238 Rho-Of-Plant (ROP) family, which have a polybasic stretch adjacent to a prenylation site (i.e. 239 a geranylgeranylation lipid modification) (91).

240 There is no precise study on the number of proteins encoded in plant genomes that might 241 contains such polybasic/hydrophobic stretches and localize to electrostatic membranes 242 through this mechanism but it is likely a general mechanism that contributes to the 243 localization of many proteins within the electrostatic membrane territory. 
246 Anionic phospholipids present different geometries (Figure 2.a). Indeed, PI and PIPs have a large

247 head group that carries the phosphorylation, whereas PA has a very small one (10). The bulk of 248 the lipid directly influences the curvature of the membrane. Thus, the conical shape of PIPs will 249 enhance positive curvature while the inverted conical shape of PA and PS will favour negative 250 curvature (Figure 2.a). Membrane curvature is of prime importance to form endocytic vesicles at 251 the PM, secretory vesicles at the TGN/EE or to internalize proteins in MVB for instance (10, 252 78). Similar than surface charges, the curvature of the membrane can also induce lipid-protein 253 interactions, via curvature sensing domain such as Bib-Amphyphisin-Rsv (BAR) domains $(7,63)$ 254 or the Amphipathic Lipid Packing Sensor (ALPS) motif, which recognizes packing defects found 255 in curved membrane. This motif can be found in association with a small stretch of positive 256 amino acids to form a so-called +ALPS motif, which allows the coincidence detection of 257 membrane charge and curvature (139). In plants, the PI4-kinase PI4K $\beta 1$ relies on such motif to 258 localize to the curved and electrostatic membrane of TGN/EEs (93).

$\rightarrow$ Include the sidebar "Lipid molecular species and lipid packing defects" here

\subsection{Clustering}

263 Proteins and lipids can also laterally segregate in membrane domains, thereby creating subcompartmentalization inside the membranes (Figure 2.c). Such clustering is often required for the protein to fulfil its biological function. While sterol has long been associated with membrane domain formation, it recently emerged that anionic lipids are also key determinant of protein 267 clustering.

268 For example, Remorins (REM) are proteins that laterally segregate at the PM in domains of 269 about $100 \mathrm{~nm}$ in size (hence termed nanodomains) (46) (Figure 5.a). REMs are involved in 270 biotic and abiotic responses, although their exact molecular function is still unknown (47). For 
example, Solanum tuberosum REMORIN group 1 isoform three (StREM1.3) limits the spreading

272 of Potato Virus X (PVX), and regulates plasmodesmata permeability (100). A combination of 273 super-resolution microscopy and biophysical experiments showed that StREM1.3 targeting into

274 nanodomains is dependent upon the presence of both sterol and PI4P and is required for 275 function (29) (Figure 5.a). PI4P is not the only anionic lipid involved protein nanoclustering into 276 membrane nanodomains. Indeed, PS stabilizes the small GTPase ROP6 into nanoclusters of 277 about 50 to $70 \mathrm{~nm}$ in diameter (91) (Figure 5.b). By contrast to REM proteins, which are 278 constitutively localized in PM nanodomains, ROP6 clustering is triggered by its activation (i.e. 279 GTP-loading) upon auxin treatment. ROP6 directly binds to PS (and other anionic lipids) via a 280 polybasic region located next to its geranylgeranylation site (91). ROP6 localization into 281 nanoclusters is essential for function since ROP6 signalling is impaired in mutant lines lacking 282 PS biosynthesis or when the PS-binding site in ROP6 is mutated. Single molecule imaging and 283 super resolution microscopy of PS biosensors at the plasma membrane suggest that PS itself, not 284 only ROP6, is present in PM nanodomains (Figure 5.b). However, unlike ROP6, PS are present in 285 PM nanodomains both in the absence or presence of auxin (91). Therefore, there are 286 prepatterned nanoplatforms of PS at the PM, which recruit GTP-loaded ROP6 upon auxin 287 treatment. Future studies are needed to uncover whether such PS nanoplatforms also contain 288 additional lipids, such as sterol or PI4P and how they are formed. The cell wall and the 289 cytoskeleton influence protein diffusion into membrane $(73,75)$. It will therefore be interesting 290 to understand the interplay between anionic lipid-induced clustering, cell wall constraints and cytosqueleton dynamics, the latter being itself regulated by anionic lipids (24).

293 All the mechanisms described above converge on the idea that anionic lipids are key 294 determinant of membrane identity, which ultimately allows the precise recruitment of proteins 295 in space and time. There are countless examples of proteins that are targeted to specific 296 membranes by anionic phospholipids either constitutively or upon certain stimuli. These examples will not be individually reviewed here. Instead, we will dedicate the rest of this 
review to one of the key questions of cell biology: how compartments maintain a specific identity despite the constant flow and exchange of proteins and lipids between compartments. We will in particular highlight how anionic lipids may contribute to membrane contact sites establishment and analyse the interplay between membrane trafficking and anionic lipids.

\section{ANIONIC LIPIDS AND ER/PM MEMBRANE CONTACT SITES IN PLANTS}

304 Several reports began to put EPCS (ER/PM MCS) at the centre of essential cellular processes including immune secretory pathway, viral movement at plasmodesmata, stabilization of the cortical ER network, endocytosis, membrane integrity, pollen, seed and root hair development $(53,64,65,90,104,134,141)$. Lipid exchange at EPCSs is still an open question in plants but a clear role for anionic lipids in EPCS establishment is documented.

In Arabidopsis, three types of EPCSs have been described (Figure 6): the S-EPCSs, which are stabilized by synaptotagmins (SYTs, othologs of the mammalian extended synaptotagmins (E-SYT)) $(6,90)$, the V-EPCSs that contain the vesicle-associated membrane-associated protein 27s (VAP27s) and VAP27-related proteins (orthologs of the mammalian VAPS and

314 yeast $\operatorname{Scs} 2)(102,132,134)$ and the PD-EPCSs, which are specifically enriched at plasmodesmata (PD) and contain MULTIPLE C2 AND TRANSMEMBRANE PROTEIN

316 (MCTP) proteins (11). Importantly, all three classes of tethering molecules, VAP27s, SYTs, 317 and MCTPs are directly interacting with anionic phospholipids and likely require these lipids 318 to interact with the PM. Nonetheless, S-EPCS and V-EPCS are spatially separated within the 319 same cell suggesting a tethering signature model where the abundance of one type of EPCS at 320 the expense of the other could vary in response to environmental or developmental cues 321 (111). Another model, the tethering arrangement model, proposes that the organization of 322 EPCS could change in response to stimuli such as lipids or calcium fluxes (26). These two 
models are not mutually exclusive.

\subsection{V-EPCS}

VAP27 are ER transmembrane proteins that are conserved among eukaryotes (138). Ten homologs of VAP27 exist in Arabidopsis (134). Three of them localize at the ER-PM contact sites: VAP27-1, VAP27-3, and VAP27-4, together with the actin binding protein NETWORKED 3C, NET3C (133) (Figure 6). VAP27-1 and VAP27-3 interact with PIPs in vitro (116). Moreover, VAP27-3 is able to interact with a lipid transfer protein from the ORP what has been described in yeast and animal cells $(3,102)$.

\subsection{S-EPCS}

335 The mammalian E-SYT1 is able in vitro to trigger glycerolipids exchanges between 336 liposomes (105). E-SYTs contain C2 domains that bind anionic phospholipids from the PM in 337 a Ca2+ depend manner (144). In favour of the tethering arrangement model, E-SYTs Ca2+ 338 binding leads to clustering of E-SYTs proteins and an increase of the interactions between E339 SYTs and PM anionic lipids (26). This results in stabilization and tightening of S-EPCS (26, $340138,144)$. In Arabidopsis, ionic stress triggers the expansion of S-EPCS in association with 341 accumulation of $\mathrm{PI}(4,5) \mathrm{P}_{2}$ at the $\mathrm{PM}$ supporting the tethering arrangement model (62). 342 Furthermore, the C2 domains of Arabidopsis SYT1 bind PS in a Ca2+ dependent-manner in 343 vitro (90) (Figure 6). The relevance of this Ca2+-dependent PS binding in vivo is currently 344 unknown but also argues in favour of the tethering arrangement model.

\subsection{PD-EPCS}

347 PD pores interconnect virtually all cells within the plant body, establishing direct PM and cytoplasmic continuity. A striking feature of PD is a strand of endoplasmic reticulum (ER) 
running through the pores and tethered tightly to PM, making PD a unique case of EPCS in eukaryotes. While SYT1 localize to PD to some extent $(64,90)$, several MCTP proteins are specifically enriched at PD and likely contribute to ER-PM tethering at the PD pore (11) (Figure 6). Molecular dynamics suggest that MCTP4 C2 domains interact with anionic lipids at the PM, in particular PS and PI4P. Accordingly, pharmacological experiments support that $\mathrm{PI} 4 \mathrm{P}$ is required for MCTP4 PD-specific localization. PDs have a specific composition in term of sterol and sphingolipid (28). By extension, an attractive hypothesis that remains to be tested, would be that the PM at PD has a unique anionic lipid signature that could contribute to MCTP PD-specific localization.

Communication between organelles and lipid exchanges occur not only at MCSs but also during vesicular trafficking. Vesicular trafficking implies exchange of membranes between compartments and anionic lipids are key actors of those exchanges. Therefore, compartments must maintain a specific lipid identity despite the constant flow and exchange of proteins and lipids that happen during vesicular trafficking. The interplay between membrane trafficking and anionic lipids will be detailed in the following section of this review.

\section{TRAFFICKING BETWEEN THE PM AND THE TGN}

\subsection{Clathrin mediated endocytosis}

368 In animal cells, it is well documented that anionic lipids play a fundamental role in 369 endocytosis. Clathrin-mediated endocytosis mainly involves $\mathrm{PI}(4,5) \mathrm{P}_{2}$ produced from PI4P by 370 PIP5P kinases (PI4P-5Ks) (81). PI(4,5) $\mathrm{P}_{2}$ allows the initiation and stabilisation of the 371 clathrin-coated pits by the recruitment of several components of the endocytic machinery 372 including the adaptor protein AP2 $(51,95)$. Moreover, PIP5Ks are directly recruited at the site 373 of endocytosis by AP2, creating a local pool of $\mathrm{PI}(4,5) \mathrm{P}_{2}$, which induces a high rate of 
374 clathrin-coated pit nucleation (81). Then, both $\mathrm{PI}(4,5) \mathrm{P}_{2}$ and $\mathrm{PI}(3,4) \mathrm{P}_{2}$ are involved in pit maturation (81).

\subsection{1. $\mathrm{PI}(4,5) \mathrm{P}_{2}$ a main actor in clathrin-mediated endocytosis}

In plant cells, the recruitment of the endocytic machinery to the PM is far less understood.

However, the role of $\mathrm{PI}(4,5) \mathrm{P}_{2}$ in endocytosis has been demonstrated indirectly using PIP5Ks over-expression and loss-of-function approaches (43, 146). Notably, a pip5k1pip5k2 (hereafter designed as pip $5 k 1 k 2$ ) mutant has abnormal clathrin-coated pits, which are less dense than in the wild type but bigger (43). This mutant, as well as PIP5K1 and PIP5K2 overexpression line, have defects in PIN endocytic trafficking, which ultimately impact both their accumulation at the PM, their polar targeting and hence auxin distribution, leading to various degrees of auxin-related developmental defects $(43,79,120)$. Over-expression of PIP5K also induces a phloem differentiation phenotype (30). PIP5K overexpression redirects the trafficking toward the vacuole leading to protein degradation and vacuole biogenesis, which in protophloem file induces vascular discontinuities (30). However, it is unclear if this increase in vacuolar trafficking is caused by an overall heightened endocytic rate at the PM or by regulation of downstream trafficking steps. In any case, $\mathrm{PI}(4,5) \mathrm{P}_{2}$ levels are constantly kept in check in the root phloem by two PM-localized $\mathrm{PI}(4,5) \mathrm{P}_{2}$ 5-phosphatases, COTYLEDON VASCULAR PATTERN 2 (CVP2) and CVP2-LIKE 1, whose double mutant shows similar phloem discontinuity as PIP5K overexpression lines (101).

394 In growing pollen tube, the exocytosis at the tip is balanced by endocytosis in the subapical

395 regions (Figure 3). These regions are marked by $\mathrm{PI}(4,5) \mathrm{P}_{2}$ locally produced by PIP5Ks and 396 restricted by degradation into diacylglycerol (DAG) by Phosphoinositide Phospholipase C 397 (PI-PLC) (17, 32) (Figure 3). Loss-of function mutants of PIP5Ks show reduced endocytosis, 398 which lead to low pollen germination rate and slow tube elongation $(41,115)$. Conversely, 
pollens overexpressing PIP5K6 show PM invagination and the formation of several tip 400 branches due to a higher rate of endocytosis at the tip of pollen tube (146). The expression of 401 a clathrin dominant negative can rescue the PIP5K6-OE phenotype indicating that $\mathrm{PI}(4,5) \mathrm{P}_{2}$ 402 synthesis enhances clathrin-mediated endocytosis (146). A similar mechanism was proposed 403 in responses to salt treatment which increases the level of $\mathrm{PI}(4,5) \mathrm{P}_{2}$ and induces the formation 404 of clathrin-coated vesicles in a PIP5K1 and PI5PK2-dependent manner $(43,56)$.

\subsubsection{PA as another regulator of clathrin-mediated endocytosis?}

407 Salt tolerance studies suggest a role for PA in endocytosis. Indeed, the level of PA is drastically increased under salt treatments (83). In addition of inducing membrane curvature, PA is able to bind clathrin heavy chain (CHC) and domain clathrin assembly proteins (77).

410 Upon salt treatment, PA production by the phospholipase PLD $\zeta 2$ induces the internalisation of PIN2 in TGN/EE (21), whereas in normal condition PA and PLDל2 are necessary for the proper cycling of PIN2 in root cells (66). This mechanism decreases the amount of PIN2 at the PM on the salt-side and induces auxin redistribution that allows the plant to redirect its root growth away from highly salt concentrated soil (21). This data suggest that PA could act as a mediator of the auxin response by activating the endocytosis of PIN proteins upon high

416 salinity conditions.

To conclude, a role for $\mathrm{PI}(4,5) \mathrm{P}_{2}$ in clathrin-mediated endocytosis is established in different cell types and developmental contexts. Nevertheless, the proteins regulated by $\mathrm{PI}(4,5) \mathrm{P}_{2}$ during endocytosis are still largely unknown. In addition, the role of other anionic lipids such as PI4P and PA in the regulation of the endocytic machinery needs to be clarified. 
424 Exocytosis is led by a tethering complex called exocyst, which is conserved among

425 eukaryotes and that targets exocytic vesicles to the PM (88). In many cases, exocytosis does

426 not occur homogenously along the PM but it is polarized. Polar exocytosis has been reported

427 in growing tip cells such as root hair and pollen tube $(8,14,67,108,118,136)$, for the polar 428 localization of auxin efflux carriers from the PIN family $(18,89,119)$, in the establishment of 429 the casparian strip (48) or the Ortmannian ring in trichomes (57 in revision, 58-59).

\subsubsection{PI $(4,5) P_{2}$ targets the Exocyst to the PM}

The exocyst complex is targeted to the PM via two subunits, SEC3 and EXO70 known to bind anionic phospholipids, in particular PI(4,5) $\mathrm{P}_{2}$. In Arabidopsis, SEC3 contains a PH domain that binds to $\mathrm{PI}(4,5) \mathrm{P}_{2}$ in vitro (8). The extensive use of biosensors and the

435 localization of lipid metabolic enzymes in plants allowed the cartography of anionic lipids in cells exhibiting polar secretion highlighting specific lipid accumulation patterns, notably for

SEC3 and/or EXO70 isoforms often correlate with the $\mathrm{PI}(4,5) \mathrm{P}_{2}$ subcellular accumulation pattern, suggesting that $\mathrm{PI}(4,5) \mathrm{P}_{2}$ contribute to their localization $(8,48,108)$. In pollen tube,

440 SEC3 and EXO70A1 partially colocalize with PI(4,5) $\mathrm{P}_{2}$ biosensors $(8,108)$. Since PIP5K mutants display slower tube elongation and reduced secretion of cell wall materials $(41,115)$, it is likely that $\mathrm{PI}(4,5) \mathrm{P}_{2}$ participates in targeting exocyst complexes to specific $\mathrm{PM}$ domains.

\subsection{2. $\mathrm{PI}(4,5) \mathrm{P}_{2}$ and $\mathrm{PA}$ : working together to confine exocytosis}

445 EXO70A1 and EXO70B1 are expressed in growing pollen tube and partially colocalize with 446 both $\mathrm{PI}(4,5) \mathrm{P}_{2}$ and PA biosensors (108). Therefore, it is possible that the coincidence 447 detection of $\mathrm{PI}(4,5) \mathrm{P}_{2}$ and $\mathrm{PA}$ in pollen tube restricts the site of exocytosis to the narrow $\mathrm{PM}$ 448 strip that contains both lipids (Figure 3). These EXO70 subunits may directly recognize each 
lipid. Alternatively, they may be sensitive to the overall membrane surface charges. One may speculate that in pollen tube, the PM membrane domain that contains a combination of both $\mathrm{PI}(4,5) \mathrm{P}_{2}$ and $\mathrm{PA}$ is highly electronegative and thereby could recruit specific EXO70 isoforms. Additional experiments are required to distinguish between these two possibilities.

\subsection{Proper secretion requires PI4P production in the TGN}

455 Exocytosis not only requires specific anionic lipids at the PM but also a regulation of the lipid composition in the TGN for proper vesicle formation. Indeed, root hair studies revealed the importance of the pool of PI4P in the TGN for polar secretion $(49,99,122,143)$. PI4Kinases, PI4K $\beta 1$ and PI4K $\beta 2$, as well as the PI4P phosphatase ROOT HAIR DEFECTIVE 4,

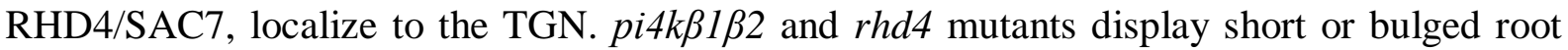
hairs $(49,99,122)$. Ultrastructural studies by electron tomography showed the presence of enlarged, likely deficient, secretory vesicles in the TGN of pi4k $\beta 1 \beta 2$ double mutant, suggesting that PI4K $\beta$ s are indeed involved in secretion (49). PI4K $\beta$ s and RHD4/SAC7 have opposite catalytic activities, yet apparently, locate to the same compartment. It is possible that 464 PI4K $\beta$ s and RHD4/SAC7 localize in different TGN subdomains. Consistent with this 465 scenario, PI4K $\beta 1$ localizes specifically in the secretory vesicle subdomain of the TGN (49). 466 According to this hypothesis, PI4Kinases could be necessary for the production of the pool of 467 PI4P at the TGN which confers the proper physical properties (mild electrostatics, high 468 curvature) necessary for secretory vesicle formation. By contrast, RHD4/SAC7 could localize 469 in a different TGN subdomain, perhaps directly connected to endocytosis. In such model, 470 RHD4/SAC7 would degrade incoming PI4P from the PM to maintain the PI4P gradient along 471 the endocytic pathway (113). 
474 The cell plate is an organelle formed from the secretion and fusion of vesicles from the TGN

475 and will form the new PM of the two daughter cells (13). A combination of clathrin-mediated

476 endocytosis and exocytosis occurs at the cell plate. During this process, the lipid composition

477 of the cell plate varies from the bulk PM. Lipid binding biosensors suggest that PI4P, PA and

478 PS accumulate and confer a highly electronegative field at the cell plate to a similar extent, or

479 even higher, than at the $\mathrm{PM}(16,93,113)$ (Figure 3). However, $\mathrm{PI}(4,5) \mathrm{P}_{2}$ is excluded from the

480 cell plate during its creation and extension and appears at the edges of the cell plate just

481 before its full attachment to the $\mathrm{PM}(16,113,127)$. Knowing that $\mathrm{PI}(4,5) \mathrm{P}_{2}$ is a major

482 regulator of both exocytosis and endocytosis, it is therefore likely that specific trafficking

483 mechanisms occur at the cell plate compared to the bulk PM, which have yet to be uncovered.

484 This difference of membrane composition between the bulk PM and the cell plate is crucial

485 for proper cell division. Indeed, several mutants for enzymes involved in anionic lipid

486 homeostasis show cell plate and/or cytokinesis phenotypes. Those include mutants for PI4P

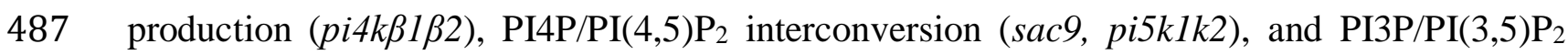

488 interconversion (fablafablb, fra7/sacl) $(49,120,131,137,148)$. Some of these enzymes

489 directly act at the cell plate. For instance, PI4K $\beta 1$ localizes at the cell plate and the absence of

490 PI4K $\beta 1$ and PI4K $\beta 2$ directly affect the vesicular trafficking along phragmoplast microtubules

491 (68). However, others might act indirectly on cell division, such as FORMATION OF

APLOID AND BINUCLEATE CELLS 1, FAB1A and FAB1B proteins, that do not localize

493 at the cell plate $(36,137)$. It is therefore likely that the fablafablb cell plate phenotype results

494 from a broad alteration of membrane trafficking. We refer the reader to a recent review on

495 this subject, which details the potential involvement of each anionic lipid during cytokinesis

496 (13). 
Fungi infection and mycorrhizal symbiosis induce PM reorganization, which include lipid patterning at the PM with likely consequences on both endocytosis and exocytosis.

501

\subsubsection{Periarbuscular membrane}

503 During the endosymbiotic association of roots with arbuscular mycorrhizal (AM) fungi in $M$.

504 truncatulla, the fungus develops intracellular branched hyphae called arbuscules (44). In 505 response, the root cortical cells form a new membrane in continuity with the PM called 506 periarbuscular membrane (PAM) (31). The PAM envelops the hyphal branches and is thought 507 to be generated by polar exocytosis. Indeed, subunit of the exocyst complex and SNARE 508 proteins known to be involved in exocytosis and membrane fusion are required for the PAM 509 formation $(31,145)$. By analogy to tip growing cells, anionic lipids might regulate this 510 particular case of polarized secretion. So far, only the pattern of localization of PI4P, $511 \mathrm{PI}(4,5) \mathrm{P}_{2}$ and PA were documented during AM interaction (44) (Figure 3). Both PI4P and PA 512 are found in the PAM to similar extent than the PM. However, in the trunk part of hyphal 513 branches, discrete regions where $\mathrm{PI}(4,5) \mathrm{P}_{2}$, PI4P and $\mathrm{PA}$ reporters co-accumulate can be 514 observed (44). This accumulation could correspond to an accumulation of membranes before 515 formation of small bulges. Furthermore, anionic lipids accumulation could influence 516 membrane curvature and thereby physically contribute to the establishment of the PAM.

\subsubsection{Extra invasive hyphal membrane}

519 Using another model of fungi-plant interactions, it was recently shown in Arabidopsis

520 infected by Colletotrichum that extra invasive hyphal membrane (EIHM), which is formed in 521 continuity with the PM, strongly accumulates PI(4,5) $\mathrm{P}_{2}$ and PIP5K3 all along the membrane

522 (Figure 3), as well as a subunit of the exocyst complex and SNARE proteins $(39,109,110)$.

523 This accumulation of $\mathrm{PI}(4,5) \mathrm{P}_{2}$ seems to be specific to Colletotrichum $(110)$. $\mathrm{PI}(4,5) \mathrm{P}_{2}$ 
524 production by PIP5K at the EIHM could increase polar exocytosis thereby allowing the

525 formation of the EIHM and thus the successful infection by the fungi.

527 All together, these recent data suggest that anionic lipid patterns are modified in response to 528 fungi and allow the symbiosis or infection to take place, possibly by induction of polar 529 exocytosis to the membrane that surround the hypha.

530

\section{ANIONIC LIPIDS IN VACUOLAR TRAFFICKING}

Most membrane-associated proteins are degraded in the vacuole. To reach the vacuole, PM proteins are first polyubiquitinated, which induces endocytosis and targeting to TGN/EEs (19). Then TGN/EEs mature into LE/MVBs. During this maturation, endosomal-sortingcomplex-required-for-transport (ESCRT) internalizes membrane proteins into intraluminal vesicles. Once the external membrane of MVB fuses with the tonoplast, the intraluminal vesicles containing the proteins are released into the vacuole and degraded. Non-ubiquitinated cargo can be retrieved in MVBs by the action of the retromer complex that antagonize the ESCRT complex (86).

\subsection{From PI4P to PI3P and PI(3,5)P2: PIP cascade leading to protein sorting}

542 One of the main characteristics of PIPs is to be quickly interconverted by kinases and 543 phosphatases (Figure 1.c). This is well illustrated along the road leading to protein 544 degradation into the vacuole in which the different maturation steps correlate with a PIP 545 interconversion (Figure 7). PI(4,5) $\mathrm{P}_{2}$ from the $\mathrm{PM}$ is converted into PI4P by 5-phosphatases 546 either in TGN/EEs or during the endocytic process. At the surface of TGN/EEs, PI4P is 547 gradually replaced by PI3P through the combined action of 4-phosphatases (e.g. RHD4/SAC7 548 in root hair) and the PI3K, VPS34. Some MVBs exhibit $\mathrm{PI}(3,5) \mathrm{P}_{2}$ due to the action of the 
PI3P5K from the FAB1 family $(36,38,112)$ (Figure 7). The acquisition of PI3P is essential

550 for protein sorting. Indeed, FYVE1/FREE1, a component of the ESCRT complex that binds

551 ubiquitinated cargo, localizes in the MVB by interacting with PI3P (22, 55). Similarly, 552 SORTING NEXIN 1 (SNX1), a component of the retromer complex also localizes at the 553 MVB through interactions with $\mathrm{PI} 3 \mathrm{P}$ and/or $\mathrm{PI}(3,5) \mathrm{P}_{2}(98)$. In addition, $\mathrm{PI} \mathrm{P}$ and/or $\mathrm{PI}(3,5) \mathrm{P}_{2}$ 554 also mediate the association of endosomes with the cytoskeleton, since the loss of FAB1 555 releases MVBs from cortical microtubules $(35,36)$ and SNX1 interact with the microtubule regulator CYTOPLASMIC LINKER ASSOCIATED PROTEIN, CLASP (1).

\subsection{Autophagosome and protein degradation}

559 Cytosolic cargoes can also be degraded by autophagy (20). In this process, cargoes are 560 encapsulated by a double membrane to form autophagosomes. The outer membrane of the 561 autophagosomes fuses with the tonoplast and the internal membrane, together with its luminal 562 content, is subsequently degraded in the vacuole (20). The autophagosome is initiated by the 563 formation of a phagophore that will expand, surround the cargoes and close. AuTophaGy564 related (ATG) proteins were first discovered by genetic screens in yeast but most of the 565 actors of autophagy are conserved among eukaryotes (20). In all eukaryotes, PI3P is critical 566 for autophagy. In animal and yeast, PI3P is produced at the initiation step of phagophore 567 formation by a PI3K complex that contains the PI3K VPS34 (20). A member of the complex, 568 ATG14, targets the PI3K complex to the phagophore (15). Furthermore, the production of $569 \mathrm{PI} 3 \mathrm{P}$ is essential to recruit and stabilize auptophagy-related proteins at the membrane of the 570 phagophore. In addition, the geometry of the head group of PI3P could also directly help the 571 curvature of the phagophore. In plants, the precise dynamics of PI3P during autophagy is not 572 well understood. Furthermore, ATG14 does not exist, which raises the question of the 573 recruitment of the PI3K complex at the phagophore (20). Yet, inhibitor of PI3Ks blocks 
autophagy in plants (142), suggesting that, like in other eukaryotes, PI3P is important for this

575 process.

576

577 In addition to stabilizing ATG-related protein at the membrane of the phagophore, several 578 plant-specific proteins, involved in autophagy, directly interact with phosphoinositides. SRC579 HOMOLOGY3-CONTAINING-PROTEINs SH3P2 and SH3P3 are two BAR-domain 580 containing proteins that localize at the phagophore (149). As BAR domains can induce 581 membrane curvature, they may participate in the membrane shaping of the phagophore. 582 SH3P2 interacts with PI3P (and other phosphoinositides) and co-localize with ATG8 in the 583 phagophore in a VPS34-dependent manner (149). SH3P2 interacts with ATG8 and the PI3K 584 complex. fyvel/freel mutant presents autophagy-related phenotype with accumulation of 585 autophagic structures $(22,149)$. FYVE1/FREE1 interacts with ATG8, the PI3K complex and 586 SH3P2 (23). As such, FYVE1/FREE1 may play a role in phagophore maturation or closing. 587 Another hypothesis argues for an indirect role of the ESCRT and retromer complexes in 588 autophagy. Indeed, SNARE proteins, necessary for autophagosome fusion with the vacuole, 589 need to be properly sorted to the tonoplast. It is therefore possible that the fyvel/free 1 590 phenotype results, at least in part, of a miss sorting of SNARE proteins (50).

\subsection{PIPs and vacuole morphology}

593 Mutant for the retromer and the ESCRT complexes present small unfused vacuoles (22, 23, $59445,54,55)$. This phenotype is also found with the loss-of- function of VTI11, a SNARE 595 protein involved in the docking and fusion of MVB with the tonoplast (124). It is suggested 596 that the PI3P-dependent protein sorting is important for the proper localization of SNARE 597 proteins and thus vacuole fusion. Moreover, the vacuole phenotype of vtill mutant can be 598 rescued by inhibition of PI3K activity suggesting a role of PI3P in fusion of homeotic 
599

600

601

602

603

604

605

606

607

608

609

610

611

612

613

614

615

616

617

618

619

620

621

622

vacuoles (147). The homotypic fusion and vacuolar protein sorting (HOPS) tethering complex is required for vacuole morphology (12). VACUOLAR PROTEIN SORTING33 (VPS33) and VPS45, two HOPS complex members, binds phosphoinositide in vitro and localize to the vacuole in a PI3P-dependent manner (12). SAC2, SAC3, SAC4 and SAC5 are likely $\mathrm{PI}(3,5) \mathrm{P}_{2}$ 5-phosphatases that localize at the tonoplast and could hydrolyse the incoming $\mathrm{PI}(3,5) \mathrm{P}_{2}$ from MVB into PI3P (87) (Figure 7). Loss-of-function and overexpression of these SAC proteins lead to abnormal vacuolar morphology consistent with deficit in vacuole fusion due to lack of PI3P (87). PI4P is also involved in the morphological changes of the vacuole that are regulated by auxin in the root $(69,106)$. However, the molecular mechanism connecting PI4P to vacuole morphology are so far unknown.

\section{CONCLUSIONS, PERSPECTIVES AND FUTURE CHALLENGES}

Many aspects of the role and operation of anionic lipids along the endomembrane system are conserved among eukaryotes, including many of the enzymes. However, the anionic lipid distribution of plant cells present several specificities compare to yeast or animal cells, starting with the high and low abundance of PI4P and $\mathrm{PI}(4,5) \mathrm{P}_{2}$ at the $\mathrm{PM}$, respectively. Thus, the establishment of the anionic lipid distribution in plant is still not fully understood. Moreover, the mechanisms by which this distribution is maintained despite the constant exchanges of membrane between compartments due to vesicular trafficking must be explored.

This includes the coupling between the enzymes that produce anionic lipids and the ones that convert or degrade them. Additionally, links or compensatory effects could exist between the different lipid pathways at the level of enzymatic activity, membrane recruitment or expression. Recently, MCS appeared as a new path of lipids exchanges independent from 
623 vesicular trafficking. The mechanisms, the nature of the exchanged lipids as well as relevance

624 for the cell and plant life remains to be fully appreciated.

625 The role of anionic lipids in the regulation of membrane trafficking has been clearly 626 established in all eukaryotes. The sorting of many of the proteins involved in membrane 627 trafficking depends on anionic lipids. At the same time, many of the proteins involved in 628 anionic lipids pathways are membrane-associated proteins. Thus, their sorting depends of 629 vesicular trafficking. The constant flow of membrane trafficking and the interconnections 630 between lipids and protein sorting make it difficult to sort the direct and indirect 631 consequences of lipid perturbation using loss-of-function strategy only. The situation is 632 further amplified by the fact that anionic phospholipids are highly interconnected, and 633 perturbation of one lipid is likely to impact others. Similarly, although anionic lipids are of 634 major importance for many developmental and physiological responses, including stress, 635 cytokinesis or cell differentiation, the chain of events explaining the observed phenotype is 636 often not solved. New live imaging and inducible perturbation technics to study lipid 637 dynamics in its physiological context are therefore urgently needed in order to untangle direct 638 from indirect effects of anionic phospholipids. Similarly, it will also be key to uncover and 639 functionally characterized anionic lipid effectors (i.e. proteins that interact with anionic 640 phospholipids for function and localization) as most of them are plant specific proteins. 
- Summary Points list: highlight the central points of your review (as many as 8), in complete sentences; insert above the Acknowledgments and/or Literature Cited section

1. Anionic lipids influence the physicochemical properties of cell membranes, such as their surface charges, curvature, packing and nanoscale organization.

2. Anionic lipids define an "electrostatic membrane territory" in plants, which corresponds to post-Golgi membranes.

3. Enrichment of specific anionic lipid combinaison in different compartments participates to the establishment of organelle identity and the specific recruitment of proteins.

4. The distribution of anionic lipids inside a membrane is not homogeneous in term of lateral segregation and membrane leaflet accumulation. Anionic lipids, together with other lipids and proteins, organize in nanoclusters and are mostly oriented toward the cytosolic leaflet.

5. Most of the enzymes related to anionic lipids are conserved among eukaryotes, while anionic lipid effectors tend to be plant specific.

6. Anionic lipids can be transported either via vesicular trafficking or via lipid transport proteins at MCS. Conversely, anionic lipids are themselves master regulators of both vesicular trafficking and MCS.

7. Phosphoinositide kinases and phosphatases allow the fast production and interconversion of PIP, which ensure relatively stable patterns of phosphoinositide subcellular accumulation despite the constant flow of anionic lipids between organelles.

8. Anionic lipid repartition is a dynamic feature of the cell and can change according to its physiological (infection, symbiosis, stress...) or developmental (polar growth, cytokinesis, aperture establishment, casparian strip formation, trichrome...) status. 
- $\square$ Future Issues list: note where research may be headed (as many as 8), in complete sentences; insert above the Acknowledgments and/or Literature Cited section

1. How the cell maintains the lipid homeostasis at both cellular and developmental levels? Addressing this question will require a deep understanding on how lipid production and degradation/consumption are coupled and regulated, and how each anionic lipid may work in functionally and physically independent pools within the same cells.

2. Most enzymes involved in anionic lipid homeostasis have been described, but how their activity is regulated is still unknown. The role of post-translational modifications (e.g. phosphorylation) has been spear headed by Ingo Heilmann's group and this is an area of research that is likely to expand in the future $(33,80)$.

3. It is difficult to determine the direct or indirect consequences of the perturbation of an anionic lipid in a given compartment. A key future research direction will be to address what is the chain of events linking defects in anionic lipids to the observed cellular or whole plant phenotypes? One of the key to unlock such question will be the development of fast inducible systems to perturb anionic phospholipids.

4. What are the feedbacks and crosstalk between anionic lipids and the other membrane lipids, including phospholipids, sterol and sphingolipids? In particular, what is the importance of the membrane bilayer asymmetry in the way lipid pools contact and regulate each other, and what is the contribution of the cell wall/PM/cytoskeleton continuum in lipid interactions across the membrane bilayer?

5. Compartment morphodynamics is a highly complex system. Understanding how anionic phospholipids contribute to membrane morphodynamics will require the development of minimal membrane systems in vitro and computer simulation together with continued efforts in molecular genetics and cellular biology.

6. Anionic lipids are key molecules for membrane contact sites, yet the importance and mechanisms of MCS formation, regulation and function are still largely enigmatic in plants. It will be particularly interesting to investigate membrane contacts which include plant specific organelles, such as the chloroplast, tonoplast, plasmodesmata or TGN.

7. The development of anionic lipid sensors compatible with live imaging and superresolution microscopy techniques has allowed analysing the function of anionic lipids with unprecedented spatial and temporal resolution. However, given the current technical revolution in the field of super-resolution microscopy, we expect this line of research to rapidly grow in the future.

8. A bottleneck in the field is our ability to biochemically detect the different anionic phospholipid molecular species with subcellular resolution. Efforts should be given to improve the current methods of anionic lipid identification by mass spectrometry and cellular fractionation. 
Acknowledgement: Research on phospholipids in Y.J. lab is supported by the French National Research Agency: ANR caLIPSO (ANR-18-CE13-0025-02) and ANR STAYINGTIGHT (ANR-18-CE13-0016-02). L.N. is funded by a $\mathrm{PhD}$ fellowships from the French Ministry of Higher Education.

720 1. Ambrose C, Ruan Y, Gardiner J, Tamblyn LM, Catching A, et al. 2013. CLASP interacts with sorting nexin 1 to link microtubules and auxin transport via PIN2 recycling in Arabidopsis thaliana. Dev. Cell. 24(6):649-59

2. Antignani V, Klocko AL, Bak G, Chandrasekaran SD, Dunivin T, Nielsen E. 2015. Recruitment of PLANT U-BOX13 and the PI4K $\beta 1 / \beta 2$ Phosphatidylinositol-4 Kinases by the Small GTPase RabA4B Plays Important Roles during Salicylic Acid-Mediated Plant Defense Signaling in Arabidopsis. Plant Cell. 27(1):243-61

3. Antonny B, Bigay J, Mesmin B. 2018. The Oxysterol-Binding Protein Cycle: Burning Off PI(4)P to Transport Cholesterol. Annu. Rev. Biochem. 87(1):809-37

4. Azouaoui H, Montigny C, Dieudonné T, Champeil P, Jacquot A, et al. 2017. High phosphatidylinositol 4-phosphate (PI4P)-dependent ATPase activity for the Drs2pCdc50p flippase after removal of its $\mathrm{N}$ - and C-terminal extensions. J. Biol. Chem. 292(19):7954-70

5. Barbosa ICR, Shikata H, Zourelidou M, Heilmann M, Heilmann I, Schwechheimer C. 2016. Phospholipid composition and a polybasic motif determine D6 PROTEIN KINASE polar association with the plasma membrane and tropic responses. Development. dev.137117

6. Bayer EM, Sparkes I, Vanneste S, Rosado A. 2017. From shaping organelles to signalling platforms: the emerging functions of plant ER-PM contact sites. Curr. Opin. Plant Biol. 40:89-96

7. Bigay J, Antonny B. 2012. Curvature, lipid packing, and electrostatics of membrane organelles: defining cellular territories in determining specificity. Dev. Cell. 23(5):886-95

8. Bloch D, Pleskot R, Pejchar P, Potocký M, Trpkošová P, et al. 2016. Exocyst SEC3 and Phosphoinositides Define Sites of Exocytosis in Pollen Tube Initiation and Growth. Plant Physiol. 172(2):980-1002

9. Botella C, Sautron E, Boudiere L, Michaud M, Dubots E, et al. 2016. ALA10, a Phospholipid Flippase, Controls FAD2/FAD3 Desaturation of Phosphatidylcholine in the ER and Affects Chloroplast Lipid Composition in Arabidopsis thaliana. Plant Physiol. 170(3):1300-1314

10. Boutté Y, Moreau P. 2014. Modulation of endomembranes morphodynamics in the secretory/retrograde pathways depends on lipid diversity. Curr. Opin. Plant Biol. 22:22-29

11. Brault ML, Petit JD, Immel F, Nicolas WJ, Glavier M, et al. 2019. Multiple C2 domains and transmembrane region proteins (MCTPs) tether membranes at plasmodesmata. EMBO Rep. 0(0):e47182

12. Brillada C, Zheng J, Krüger F, Rovira-Diaz E, Askani JC, et al. 2018. Phosphoinositides control the localization of HOPS subunit VPS41, which together with VPS33 mediates vacuole fusion in plants. Proc. Natl. Acad. Sci., p. 201807763

13. Caillaud M-C. 2019. Anionic Lipids: A Pipeline Connecting Key Players of Plant Cell Division. Front. Plant Sci. 10:

14. Cole RA, Synek L, Zarsky V, Fowler JE. 2005. SEC8, a Subunit of the Putative Arabidopsis Exocyst Complex, Facilitates Pollen Germination and Competitive Pollen 

tethering and fusion of autophagosomes to endolysosomes. Nature. 520(7548):563-66 16. Doumane M, Lionnet C, Bayle V, Jaillais Y, Caillaud M-C. 2017. Automated Tracking of Root for Confocal Time-lapse Imaging of Cellular Processes. Bio-Protoc. 7(8): 17. Dowd PE, Coursol S, Skirpan AL, Kao T, Gilroy S. 2006. Petunia phospholipase c1 is involved in pollen tube growth. Plant Cell. 18(6):1438-53 18. Drdová EJ, Synek L, Pečenková T, Hála M, Kulich I, et al. 2013. The exocyst complex contributes to PIN auxin efflux carrier recycling and polar auxin transport in Arabidopsis. Plant J. 73(5):709-19 19. Dubeaux G, Vert G. 2017. Zooming into plant ubiquitin-mediated endocytosis. Curr. Opin. Plant Biol. 40:56-62

20. Enrique Gomez R, Joubès J, Valentin N, Batoko H, Satiat-Jeunemaître B, Bernard A. 2018. Lipids in membrane dynamics during autophagy in plants. J. Exp. Bot. 69(6):128799

21. Galvan-Ampudia CS, Julkowska MM, Darwish E, Gandullo J, Korver RA, et al. 2013. Halotropism Is a Response of Plant Roots to Avoid a Saline Environment. Curr. Biol. 23(20):2044-50

22. Gao C, Luo M, Zhao Q, Yang R, Cui Y, et al. 2014. A Unique Plant ESCRT Component, FREE1, Regulates Multivesicular Body Protein Sorting and Plant Growth. Curr. Biol. 24(21):2556-63

23. Gao C, Zhuang X, Cui Y, Fu X, He Y, et al. 2015. Dual roles of an Arabidopsis ESCRT component FREE1 in regulating vacuolar protein transport and autophagic degradation. Proc. Natl. Acad. Sci. U. S. A. 112(6):1886-91

24. Gerth K, Lin F, Menzel W, Krishnamoorthy P, Stenzel I, et al. 2017. Guilt by Association: A Phenotype-Based View of the Plant Phosphoinositide Network. Annu. Rev. Plant Biol. 68(1):349-74

25. Ghosh R, de Campos MKF, Huang J, Huh SK, Orlowski A, et al. 2015. Sec14-nodulin proteins and the patterning of phosphoinositide landmarks for developmental control of membrane morphogenesis. Mol. Biol. Cell. 26(9):1764-81

26. Giordano F, Saheki Y, Idevall-Hagren O, Colombo SF, Pirruccello M, et al. 2013. $\mathrm{PI}(4,5) \mathrm{P}(2)$-dependent and $\mathrm{Ca}(2+)$-regulated ER-PM interactions mediated by the extended synaptotagmins. Cell. 153(7):1494-1509

27. Gomès E, Jakobsen MK, Axelsen KB, Geisler M, Palmgren MG. 2000. Chilling Tolerance in Arabidopsis Involves ALA1, a Member of a New Family of Putative Aminophospholipid Translocases. Plant Cell. 12(12):2441-53

28. Grison MS, Brocard L, Fouillen L, Nicolas W, Wewer V, et al. 2015. Specific membrane lipid composition is important for plasmodesmata function in Arabidopsis. Plant Cell. 27(4):1228-50

29. Gronnier J, Crowet J-M, Habenstein B, Nasir MN, Bayle V, et al. 2017. Structural basis for plant plasma membrane protein dynamics and organization into functional nanodomains. eLife. 6:

30. Gujas B, Cruz TMD, Kastanaki E, Vermeer JEM, Munnik T, Rodriguez-Villalon A. 2017. Perturbing phosphoinositide homeostasis oppositely affects vascular differentiation in Arabidopsis thaliana roots. Development. 144(19):3578-89

31. Harrison MJ, Ivanov S. 2017. Exocytosis for endosymbiosis: membrane trafficking pathways for development of symbiotic membrane compartments. Curr. Opin. Plant Biol. 38:101-8

32. Helling D, Possart A, Cottier S, Klahre U, Kost B. 2006. Pollen tube tip growth 
depends on plasma membrane polarization mediated by tobacco PLC3 activity and endocytic membrane recycling. Plant Cell. 18(12):3519-34 33. Hempel F, Stenzel I, Heilmann M, Krishnamoorthy P, Menzel W, et al. 2017. MAPKs Influence Pollen Tube Growth by Controlling the Formation of Phosphatidylinositol 4,5-Bisphosphate in an Apical Plasma Membrane Domain. Plant Cell. 29(12):3030-50

34. Hirano T, Konno H, Takeda S, Dolan L, Kato M, et al. 2018. PtdIns(3,5)P 2 mediates root hair shank hardening in Arabidopsis. Nat. Plants. 4(11):888

35. Hirano T, Matsuzawa T, Takegawa K, Sato MH. 2011. Loss-of-Function and Gainof-Function Mutations in FAB1A/B Impair Endomembrane Homeostasis, Conferring Pleiotropic Developmental Abnormalities in Arabidopsis. Plant Physiol. 155(2):797-807 36. Hirano T, Munnik T, Sato MH. 2015. Phosphatidylinositol 3-phosphate 5-kinase, FAB1/PIKfyve mediates endosome maturation to establish endosome-cortical microtubule interaction in Arabidopsis. Plant Physiol. pp.01368.2015

37. Hirano T, Munnik T, Sato MH. 2016. Inhibition of phosphatidylinositol 3,5bisphosphate production has pleiotropic effects on various membrane trafficking routes in Arabidopsis. Plant Cell Physiol., p. pcw164 38. Hirano T, Stecker K, Munnik T, Xu H, Sato MH. 2017. Visualization of phosphatidylinositol 3,5-bisphosphate dynamics by tandem ML1N-based fluorescent protein probe in Arabidopsis. Plant Cell Physiol. Entry mode-dependent function of an indole glucosinolate pathway in Arabidopsis for nonhost resistance against anthracnose pathogens. Plant Cell. 22(7):2429-43 40. Huang J, Ghosh R, Tripathi A, Lönnfors M, Somerharju P, Bankaitis VA. 2016. Twoligand priming mechanism for potentiated phosphoinositide synthesis is an evolutionarily conserved feature of Sec14-like phosphatidylinositol and phosphatidylcholine exchange proteins. Mol. Biol. Cell. 27(14):2317-30

41. Ischebeck T, Stenzel I, Heilmann I. 2008. Type B Phosphatidylinositol-4Phosphate 5-Kinases Mediate Arabidopsis and Nicotiana tabacum Pollen Tube Growth by Regulating Apical Pectin Secretion. Plant Cell. 20(12):3312-30 42. Ischebeck T, Stenzel I, Hempel F, Jin X, Mosblech A, Heilmann I. 2011. Phosphatidylinositol-4,5-bisphosphate influences Nt-Rac5-mediated cell expansion in pollen tubes of Nicotiana tabacum. Plant J. 65(3):453-68

43. Ischebeck T, Werner S, Krishnamoorthy P, Lerche J, Meijón M, et al. 2013. Phosphatidylinositol 4,5-Bisphosphate Influences PIN Polarization by Controlling Clathrin-Mediated Membrane Trafficking in Arabidopsis. Plant Cell. 25(12):4894-4911

44. Ivanov S, Harrison MJ. 2019. Accumulation of phosphoinositides in distinct regions of the periarbuscular membrane. New Phytol. 221(4):2213-27

45. Jaillais Y, Santambrogio M, Rozier F, Fobis-Loisy I, Miège C, Gaude T. 2007. The retromer protein VPS29 links cell polarity and organ initiation in plants. Cell. 130(6):1057-70

46. Jarsch IK, Konrad SSA, Stratil TF, Urbanus SL, Szymanski W, et al. 2014. Plasma Membranes Are Subcompartmentalized into a Plethora of Coexisting and Diverse Microdomains in Arabidopsis and Nicotiana benthamiana. Plant Cell. 26(4):1698-1711 47. Jarsch IK, Ott T. 2011. Perspectives on remorin proteins, membrane rafts, and their role during plant-microbe interactions. Mol. Plant-Microbe Interact. MPMI. 24(1):712

48. Kalmbach L, Hématy K, De Bellis D, Barberon M, Fujita S, et al. 2017. Transient cell-specific EX070A1 activity in the CASP domain and Casparian strip localization. Nat. 
49. Kang B-H, Nielsen E, Preuss ML, Mastronarde D, Staehelin LA. 2011. Electron Tomography of RabA4b- and PI-4K $\beta 1$-Labeled Trans Golgi Network Compartments in Arabidopsis. Traffic. 12(3):313-29

50. Katsiarimpa A, Kalinowska K, Anzenberger F, Weis C, Ostertag M, et al. 2013. The deubiquitinating enzyme AMSH1 and the ESCRT-III subunit VPS2.1 are required for autophagic degradation in Arabidopsis. Plant Cell. 25(6):2236-52

51. Kelly BT, Graham SC, Liska N, Dannhauser PN, Höning S, et al. 2014. AP2 controls clathrin polymerization with a membrane-activated switch. Science. 345(6195):459-63 52. KF de Campos M, Schaaf G. 2017. The regulation of cell polarity by lipid transfer proteins of the SEC14 family. Curr. Opin. Plant Biol. 40:158-68

53. Kim H, Kwon H, Kim S, Kim MK, Botella MA, et al. 2016. Synaptotagmin 1 Negatively Controls the Two Distinct Immune Secretory Pathways to Powdery Mildew Fungi in Arabidopsis. Plant Cell Physiol. 57(6):1133-41

54. Kleine-Vehn J, Leitner J, Zwiewka M, Sauer M, Abas L, et al. 2008. Differential degradation of PIN2 auxin efflux carrier by retromer-dependent vacuolar targeting. Proc. Natl. Acad. Sci. U. S. A. 105(46):17812-17 55. Kolb C, Nagel M-K, Kalinowska K, Hagmann J, Ichikawa M, et al. 2015. FYVE1 is essential for vacuole biogenesis and intracellular trafficking in Arabidopsis. Plant Physiol. 167(4):1361-73 association of phosphatidylinositol 4,5-bisphosphate with clathrin-coated vesicles in plants. Biochem. J. 415(3):387-99

57. Kubátová Z, Pejchar P, Potocký M, Sekereš J, Žárský V, Kulich I. 2019. Arabidopsis trichome contains two plasma membrane 3 domains with different lipid composition which 4 attract distinct EXO70 subunits. Int J Mol Sci, p. 15

58. Kulich I, Vojtíková Z, Glanc M, Ortmannová J, Rasmann S, Žárský V. 2015. Cell wall maturation of Arabidopsis trichomes is dependent on exocyst subunit EXO70H4 and involves callose deposition. Plant Physiol. 168(1):120-31

59. Kulich I, Vojtíková Z, Sabol P, Ortmannová J, Neděla V, et al. 2018. Exocyst Subunit EX070H4 Has a Specific Role in Callose Synthase Secretion and Silica Accumulation1[OPEN]. Plant Physiol. 176(3):2040-51

60. Kusano H, Testerink C, Vermeer JEM, Tsuge T, Shimada H, et al. 2008. The Arabidopsis Phosphatidylinositol Phosphate 5-Kinase PIP5K3 Is a Key Regulator of Root Hair Tip Growth. Plant Cell. 20(2):367-80

61. Lee BH, Weber ZT, Zourelidou M, Hofmeister BT, Schmitz RJ, et al. 2018. Arabidopsis Protein Kinase D6PKL3 Is Involved in the Formation of Distinct Plasma Membrane Aperture Domains on the Pollen Surface. Plant Cell. 30(9):2038-56

62. Lee E, Vanneste S, Pérez-Sancho J, Benitez-Fuente F, Strelau M, et al. 2019. Ionic stress enhances ER-PM connectivity via phosphoinositide-associated SYT1 contact site expansion in Arabidopsis. Proc. Natl. Acad. Sci. 116(4):1420-29

63. Lemmon MA. 2008. Membrane recognition by phospholipid-binding domains. Nat. Rev. Mol. Cell Biol. 9(2):99-111

64. Levy A, Zheng JY, Lazarowitz SG. 2015. Synaptotagmin SYTA forms ER-plasma membrane junctions that are recruited to plasmodesmata for plant virus movement. Curr. Biol. CB. 25(15):2018-25

65. Lewis JD, Lazarowitz SG. 2010. Arabidopsis synaptotagmin SYTA regulates endocytosis and virus movement protein cell-to-cell transport. Proc. Natl. Acad. Sci. U. S. A. 107(6):2491-96 

Required for Auxin Response. Plant Cell. 19(1):281-95

67. Li Y, Tan X, Wang M, Li B, Zhao Y, et al. 2017. Exocyst subunit SEC3A marks the germination site and is essential for pollen germination in Arabidopsis thaliana. Sci. Rep. 7:

68. Lin F, Krishnamoorthy P, Schubert V, Hause G, Heilmann M, Heilmann I. 2019. A dual role for cell plate-associated PI4K $\beta$ in endocytosis and phragmoplast dynamics during plant somatic cytokinesis. EMBO J. 38(4):

69. Löfke C, Dünser K, Scheuring D, Kleine-Vehn J. 2015. Auxin regulates SNAREdependent vacuolar morphology restricting cell size. eLife. 4:e05868

70. López-Marqués RL, Poulsen LR, Hanisch S, Meffert K, Buch-Pedersen MJ, et al. 2010. Intracellular targeting signals and lipid specificity determinants of the ALA/ALIS P4-ATPase complex reside in the catalytic ALA alpha-subunit. Mol. Biol. Cell. 21(5):791801

71. López-Marqués RL, Poulsen LR, Palmgren MG. 2012. A Putative Plant Aminophospholipid Flippase, the Arabidopsis P4 ATPase ALA1, Localizes to the Plasma Membrane following Association with a $\beta$-Subunit. PLOS ONE. 7(4):

72. Lynch DV, Steponkus PL. 1987. Plasma Membrane Lipid Alterations Associated with Cold Acclimation of Winter Rye Seedlings (Secale cereale L. cv Puma). Plant Physiol. 83(4):761-67

930 73. Martinière A, Lavagi I, Nageswaran G, Rolfe DJ, Maneta-Peyret L, et al. 2012. Cell wall constrains lateral diffusion of plant plasma-membrane proteins. Proc. Natl. Acad. Sci. U. S. A. 109(31):12805-10

74. McDowell SC, López-Marqués RL, Cohen T, Brown E, Rosenberg A, et al. 2015. Loss of the Arabidopsis thaliana P4-ATPases ALA6 and ALA7 impairs pollen fitness and alters the pollen tube plasma membrane. Front. Plant Sci. 6:197

75. McKenna JF, Rolfe DJ, Webb SED, Tolmie AF, Botchway SW, et al. 2019. The cell wall regulates dynamics and size of plasma-membrane nanodomains in Arabidopsis. Proc. Natl. Acad. Sci. 116(26):12857-62

939 76. McLaughlin S. 1989. The Electrostatic Properties of Membranes. Annu. Rev. 940 Biophys. Biophys. Chem. 18(1):113-36

941 77. McLoughlin F, Arisz SA, Dekker HL, Kramer G, de Koster CG, et al. 2013. Identification of novel candidate phosphatidic acid-binding proteins involved in the saltstress response of Arabidopsis thaliana roots. Biochem. J. 450(3):573-81

78. McMahon HT, Gallop JL. 2005. Membrane curvature and mechanisms of dynamic cell membrane remodelling. Nature. 438(7068):590-96

79. Mei Y, Jia W-J, Chu Y-J, Xue H-W. 2012. Arabidopsis phosphatidylinositol monophosphate 5-kinase 2 is involved in root gravitropism through regulation of polar auxin transport by affecting the cycling of PIN proteins. Cell Res. 22(3):581-97

80. Menzel W, Stenzel I, Helbig L-M, Krishnamoorthy P, Neumann S, et al. 2019. A PAMP-triggered MAPK-cascade inhibits phosphatidylinositol 4,5-bisphosphate production by PIP5K6 in Arabidopsis thaliana. New Phytol.

81. Mettlen M, Chen P-H, Srinivasan S, Danuser G, Schmid SL. 2018. Regulation of Clathrin-Mediated Endocytosis. Annu. Rev. Biochem. 87(1):871-96

82. Mishkind M, Vermeer JEM, Darwish E, Munnik T. 2009. Heat stress activates phospholipase D and triggers PIP accumulation at the plasma membrane and nucleus. Plant J. Cell Mol. Biol. 60(1):10-21

83. Munnik T, Meijer HJ, Ter Riet B, Hirt H, Frank W, et al. 2000. Hyperosmotic stress stimulates phospholipase D activity and elevates the levels of phosphatidic acid and 
960 84. Munnik T, Nielsen E. 2011. Green light for polyphosphoinositide signals in plants.

961 Curr. Opin. Plant Biol. 14(5):489-97

85. Nintemann SJ, Palmgren M, López-Marqués RL. 2019. Catch You on the Flip Side:

A Critical Review of Flippase Mutant Phenotypes. Trends Plant Sci. 24(5):468-78

86. Noack LC, Jaillais Y. 2017. Precision targeting by phosphoinositides: how PIs direct endomembrane trafficking in plants. Curr. Opin. Plant Biol. 40:22-33

87. Nováková P, Hirsch S, Feraru E, Tejos R, Wijk R van, et al. 2014. SAC phosphoinositide phosphatases at the tonoplast mediate vacuolar function in Arabidopsis. Proc. Natl. Acad. Sci. 111(7):2818-23 88. Novick P, Field C, Schekman R. 1980. Identification of 23 complementation groups required for post-translational events in the yeast secretory pathway. Cell. 21(1):205-15

89. Ogura T, Goeschl C, Filiault D, Mirea M, Slovak R, et al. 2019. Root System Depth in Arabidopsis Is Shaped by EXOCYST70A3 via the Dynamic Modulation of Auxin Transport. Cell. 178(2):400-412.e16

90. Pérez-Sancho J, Vanneste S, Lee E, McFarlane HE, Valle AE del, et al. 2015. The Arabidopsis Synaptotagmin1 Is Enriched in Endoplasmic Reticulum-Plasma Membrane Contact Sites and Confers Cellular Resistance to Mechanical Stresses. Plant Physiol. 168(1):132-43

91. Platre MP, Bayle V, Armengot L, Bareille J, Marquès-Bueno M del M, et al. 2019. Developmental control of plant Rho GTPase nano-organization by the lipid phosphatidylserine. Science. 364(6435):57-62

92. Platre MP, Jaillais Y. 2016. Guidelines for the use of protein domains in acidic phospholipid imaging. Methods Mol. Biol. Clifton NJ. 1376:175-94

93. Platre MP, Noack LC, Doumane M, Bayle V, Simon MLA, et al. 2018. A Combinatorial Lipid Code Shapes the Electrostatic Landscape of Plant Endomembranes. Dev. Cell. 45(4):465-480.e11

94. Pokotylo I, Kravets V, Martinec J, Ruelland E. 2018. The phosphatidic acid paradox: Too many actions for one molecule class? Lessons from plants. Prog. Lipid Res. 71:43-53

95. Posor Y, Eichhorn-Grünig M, Haucke V. 2015. Phosphoinositides in endocytosis. Biochim. Biophys. Acta BBA - Mol. Cell Biol. Lipids. 1851(6):794-804

96. Poulsen LR, López-Marqués RL, McDowell SC, Okkeri J, Licht D, et al. 2008. The Arabidopsis P4-ATPase ALA3 localizes to the golgi and requires a beta-subunit to function in lipid translocation and secretory vesicle formation. Plant Cell. 20(3):658-76 97. Poulsen LR, López-Marqués RL, Pedas PR, McDowell SC, Brown E, et al. 2015. A phospholipid uptake system in the model plant Arabidopsis thaliana. Nat. Commun. 6:7649

98. Pourcher M, Santambrogio M, Thazar N, Thierry A-M, Fobis-Loisy I, et al. 2010. Analyses of sorting nexins reveal distinct retromer-subcomplex functions in development and protein sorting in Arabidopsis thaliana. Plant Cell. 22(12):3980-91 99. Preuss ML, Schmitz AJ, Thole JM, Bonner HKS, Otegui MS, Nielsen E. 2006. A role for the RabA4b effector protein PI-4K $\beta 1$ in polarized expansion of root hair cells in Arabidopsis thaliana. J. Cell Biol. 172(7):991-98

1004 100. Raffaele S, Bayer E, Lafarge D, Cluzet S, German Retana S, et al. 2009. Remorin, a 1005 solanaceae protein resident in membrane rafts and plasmodesmata, impairs potato virus X movement. Plant Cell. 21(5):1541-55

101. Rodriguez-Villalon A, Gujas B, Wijk R van, Munnik T, Hardtke CS. 2015. Primary 
1008

1009

1010

1011

1012

1013

1014

1015

1016

1017

1018

1019

1020

1021

1022

1023

1024

1025

1026

1027

1028

1029

1030

1031

1032

1033

1034

1035

1036

1037

1038

1039

1040

1041

1042

1043

1044

1045

1046

1047

1048

1049

1050

1051

1052

1053

1054

1055

1056

root protophloem differentiation requires balanced phosphatidylinositol-4,5biphosphate levels and systemically affects root branching. Development. 142(8):143746

102. Saravanan RS, Slabaugh E, Singh VR, Lapidus LJ, Haas T, Brandizzi F. 2009. The targeting of the oxysterol-binding protein ORP3a to the endoplasmic reticulum relies on the plant VAP33 homolog PVA12. Plant J. 58(5):817-30

103. Schaaf G, Ortlund EA, Tyeryar KR, Mousley CJ, Ile KE, et al. 2008. Functional Anatomy of Phospholipid Binding and Regulation of Phosphoinositide Homeostasis by Proteins of the Sec14 Superfamily. Mol. Cell. 29(2):191-206

104. Schapire AL, Voigt B, Jasik J, Rosado A, Lopez-Cobollo R, et al. 2008. Arabidopsis Synaptotagmin 1 Is Required for the Maintenance of Plasma Membrane Integrity and Cell Viability. Plant Cell. 20(12):3374-88

105. Schauder CM, Wu X, Saheki Y, Narayanaswamy P, Torta F, et al. 2014. Structure of a lipid-bound Extended-Synaptotagmin indicates a role in lipid transfer. Nature. 510(7506):552-55

106. Scheuring D, Löfke C, Krüger F, Kittelmann M, Eisa A, et al. 2016. Actin-dependent vacuolar occupancy of the cell determines auxin-induced growth repression. Proc. Natl. Acad. Sci. 113(2):452-57

107. Scorrano L, Matteis MAD, Emr S, Giordano F, Hajnóczky G, et al. 2019. Coming together to define membrane contact sites. Nat. Commun. 10(1):1287

108. Sekereš J, Pejchar P, Šantrůček J, Vukašinović N, Žárský V, Potocký M. 2017. Analysis of Exocyst Subunit EXO70 Family Reveals Distinct Membrane Polar Domains in Tobacco Pollen Tubes. Plant Physiol. 173(3):1659-75

109. Shimada C, Lipka V, O'Connell R, Okuno T, Schulze-Lefert P, Takano Y. 2006. Nonhost resistance in Arabidopsis-Colletotrichum interactions acts at the cell periphery and requires actin filament function. Mol. Plant-Microbe Interact. MPMI. 19(3):270-79

110. Shimada TL, Betsuyaku S, Inada N, Ebine K, Fujimoto M, et al. 2019. Enrichment of Phosphatidylinositol 4,5-Bisphosphate in the Extra-Invasive Hyphal Membrane Promotes Colletotrichum Infection of Arabidopsis thaliana. Plant Cell Physiol., p. pcz058

111. Siao W, Wang P, Voigt B, Hussey PJ, Baluska F. 2016. Arabidopsis SYT1 maintains stability of cortical endoplasmic reticulum networks and VAP27-1-enriched endoplasmic reticulum-plasma membrane contact sites. J. Exp. Bot. 67(21):6161-71

112. Simon MLA, Platre MP, Assil S, van Wijk R, Chen WY, et al. 2014. A multicolour/multi-affinity marker set to visualize phosphoinositide dynamics in Arabidopsis. Plant J. Cell Mol. Biol. 77(2):322-37

113. Simon MLA, Platre MP, Marquès-Bueno MM, Armengot L, Stanislas T, et al. 2016. A PtdIns(4)P-driven electrostatic field controls cell membrane identity and signalling in plants. Nat. Plants. 2(7):nplants201689

114. Singh MK, Krüger F, Beckmann H, Brumm S, Vermeer JEM, et al. 2014. Protein delivery to vacuole requires SAND protein-dependent Rab GTPase conversion for MVBvacuole fusion. Curr. Biol. CB. 24(12):1383-89

115. Sousa E, Kost B, Malhó R. 2008. Arabidopsis Phosphatidylinositol-4Monophosphate 5-Kinase 4 Regulates Pollen Tube Growth and Polarity by Modulating Membrane Recycling. Plant Cell. 20(11):3050-64

116. Stefano G, Renna L, Wormsbaecher C, Gamble J, Zienkiewicz K, Brandizzi F. 2018. Plant Endocytosis Requires the ER Membrane-Anchored Proteins VAP27-1 and VAP273. Cell Rep. 23(8):2299-2307

117. Stenzel I, Ischebeck T, König S, Hołubowska A, Sporysz M, et al. 2008. The Type B Phosphatidylinositol-4-Phosphate 5-Kinase 3 Is Essential for Root Hair Formation in 

a member of a family of putative exocyst subunits specifically expanded in land plants, is important for polar growth and plant development. Plant J. Cell Mol. Biol. 48(1):54-72 119. Tan X, Feng Y, Liu Y, Bao Y. 2016. Mutations in exocyst complex subunit SEC6 gene impaired polar auxin transport and PIN protein recycling in Arabidopsis primary root. Plant Sci. 250:97-104

120. Tejos R, Sauer M, Vanneste S, Palacios-Gomez M, Li H, et al. 2014. Bipolar Plasma Membrane Distribution of Phosphoinositides and Their Requirement for AuxinMediated Cell Polarity and Patterning in Arabidopsis. Plant Cell. 26(5):2114-28 121. Testerink C, Munnik T. 2011. Molecular, cellular, and physiological responses to phosphatidic acid formation in plants. J. Exp. Bot. 62(7):2349-61

122. Thole JM, Vermeer JEM, Zhang Y, Gadella TWJ, Nielsen E. 2008. ROOT HAIR DEFECTIVE4 Encodes a Phosphatidylinositol-4-Phosphate Phosphatase Required for Proper Root Hair Development in Arabidopsis thaliana. Plant Cell. 20(2):381-95

123. Timcenko M, Lyons JA, Januliene D, Ulstrup JJ, Dieudonné T, et al. 2019. Structure and autoregulation of a P4-ATPase lipid flippase. Nature. 571(7765):366

124. Uemura T, Ueda T. 2014. Plant vacuolar trafficking driven by RAB and SNARE proteins. Curr. Opin. Plant Biol. 22:116-21

125. Uličná L, Paprčková D, Fáberová V, Hozák P. 2018. Phospholipids and inositol phosphates linked to the epigenome. Histochem. Cell Biol. 150(3):245-53

126. van Leeuwen W, Okrész L, Bögre L, Munnik T. 2004. Learning the lipid language of plant signalling. Trends Plant Sci. 9(8):378-84

127. van Leeuwen W, Vermeer JEM, Gadella TWJ, Munnik T. 2007. Visualization of phosphatidylinositol 4,5-bisphosphate in the plasma membrane of suspension-cultured tobacco BY-2 cells and whole Arabidopsis seedlings. Plant J. Cell Mol. Biol. 52(6):101426

128. Vermeer JEM, Thole JM, Goedhart J, Nielsen E, Munnik T, Gadella Jr TWJ. 2009. Imaging phosphatidylinositol 4-phosphate dynamics in living plant cells. Plant J. 57(2):356-72

129. Vermeer JEM, van Leeuwen W, Tobeña-Santamaria R, Laxalt AM, Jones DR, et al. 2006. Visualization of PtdIns3P dynamics in living plant cells. Plant J. Cell Mol. Biol. 47(5):687-700

130. Vincent P, Chua M, Nogue F, Fairbrother A, Mekeel H, et al. 2005. A Sec14pnodulin domain phosphatidylinositol transfer protein polarizes membrane growth of Arabidopsis thaliana root hairs. J. Cell Biol. 168(5):801-12

131. Vollmer AH, Youssef NN, DeWald DB. 2011. Unique cell wall abnormalities in the putative phosphoinositide phosphatase mutant AtSAC9. Planta. 234(5):993-1005

132. Wang P, Hawes C, Hussey PJ. 2016. Plant Endoplasmic Reticulum-Plasma Membrane Contact Sites. Trends Plant Sci. 0(0):

133. Wang P, Hawkins TJ, Richardson C, Cummins I, Deeks MJ, et al. 2014. The Plant Cytoskeleton, NET3C, and VAP27 Mediate the Link between the Plasma Membrane and Endoplasmic Reticulum. Curr. Biol. 24(12):1397-1405

134. Wang P, Richardson C, Hawkins TJ, Sparkes I, Hawes C, Hussey PJ. 2016. Plant VAP27 proteins: domain characterization, intracellular localization and role in plant development. New Phytol. 210(4):1311-26

135. Welti R, Li W, Li M, Sang Y, Biesiada H, et al. 2002. Profiling Membrane Lipids in Plant Stress Responses ROLE OF PHOSPHOLIPASE D $\alpha$ IN FREEZING-INDUCED LIPID CHANGES IN ARABIDOPSIS. J. Biol. Chem. 277(35):31994-2 
136. Wen T-J, Hochholdinger F, Sauer M, Bruce W, Schnable PS. 2005. The roothairless1 Gene of Maize Encodes a Homolog of sec3, Which Is Involved in Polar Exocytosis. Plant Physiol. 138(3):1637-43 137. Whitley P, Hinz S, Doughty J. 2009. Arabidopsis FAB1/PIKfyve Proteins Are Essential for Development of Viable Pollen. PLANT Physiol. 151(4):1812-22

138. Wu H, Carvalho P, Voeltz GK. 2018. Here, there, and everywhere: The importance of ER membrane contact sites. Science. 361(6401):eaan5835

139. $\mathrm{Xu} \mathrm{P,} \mathrm{Baldridge} \mathrm{RD,} \mathrm{Chi} \mathrm{RJ,} \mathrm{Burd} \mathrm{CG,} \mathrm{Graham} \mathrm{TR.} \mathrm{2013.} \mathrm{Phosphatidylserine}$ flipping enhances membrane curvature and negative charge required for vesicular transport. J. Cell Biol. 202(6):875-86

140. Yamaoka Y, Yu Y, Mizoi J, Fujiki Y, Saito K, et al. 2011. PHOSPHATIDYLSERINE SYNTHASE1 is required for microspore development in Arabidopsis thaliana. Plant J. 67(4):648-61

141. Yamazaki T, Kawamura Y, Minami A, Uemura M. 2008. Calcium-dependent freezing tolerance in Arabidopsis involves membrane resealing via synaptotagmin SYT1. Plant Cell. 20(12):3389-3404

142. Yang X, Bassham DC. 2015. Chapter One - New Insight into the Mechanism and Function of Autophagy in Plant Cells. In International Review of Cell and Molecular Biology, ed KW Jeon. 320:1-40. Academic Press

143. Yoo C-M, Quan L, Cannon AE, Wen J, Blancaflor EB. 2012. AGD1, a class 1 ARFGAP, acts in common signaling pathways with phosphoinositide metabolism and the actin cytoskeleton in controlling Arabidopsis root hair polarity. Plant J. Cell Mol. Biol. 69(6):1064-76

144. Yu H, Liu Y, Gulbranson DR, Paine A, Rathore SS, Shen J. 2016. Extended synaptotagmins are $\mathrm{Ca} 2+-$ dependent lipid transfer proteins at membrane contact sites. Proc. Natl. Acad. Sci. 113(16):4362-67

145. Zhang X, Pumplin N, Ivanov S, Harrison MJ. 2015. EX070I Is Required for Development of a Sub-domain of the Periarbuscular Membrane during Arbuscular Mycorrhizal Symbiosis. Curr. Biol. CB. 25(16):2189-95

146. Zhao Y, Yan A, Feijó JA, Furutani M, Takenawa T, et al. 2010. Phosphoinositides Regulate Clathrin-Dependent Endocytosis at the Tip of Pollen Tubes in Arabidopsis and Tobacco. Plant Cell. 22(12):4031-44

147. Zheng J, Han SW, Rodriguez-Welsh MF, Rojas-Pierce M. 2014. Homotypic Vacuole Fusion Requires VTI11 and Is Regulated by Phosphoinositides. Mol. Plant. 7(6):1026-40 148. Zhong R, Burk DH, Nairn CJ, Wood-Jones A, Morrison WH, Ye Z-H. 2005. Mutation of SAC1, an Arabidopsis SAC domain phosphoinositide phosphatase, causes alterations in cell morphogenesis, cell wall synthesis, and actin organization. Plant Cell. 17(5):144966

149. Zhuang X, Wang H, Lam SK, Gao C, Wang X, et al. 2013. A BAR-domain protein SH3P2, which binds to phosphatidylinositol 3-phosphate and ATG8, regulates autophagosome formation in Arabidopsis. Plant Cell. 25(11):4596-4615 
- Reference Annotations: brief (15 words maximum) explanation of citations' importance (as many as 10); insert below the Literature Cited section

1. Brault et al., 2019: Proposes MCTP proteins, a class of putative PS/PI4P binding proteins, as tethering factors at PD-EPCS.

2. Gronnier et al., 2017: Describes how anionic lipids and sterol influence REMORIN conformation, localization and activity by forming nanoclusters.

3. Platre el al., 2019: Visualizes and functionally characterizes nanoclusters of anionic lipids (i.e. PS) in live plant cells.

4. Lin et al., 2019: Establishes that PI4P production at the cell plate contributes to cytokinesis and phragmoplast dynamics.

5. Kang et al., 2011: Uses electron tomography to reveal distinct TGN subdomains with PI4Kß1 highlighting the secretory vesicles subdomain.

6. Lee et al., 2019: Proposes that S-EPCS dynamics is regulated by $\mathrm{PI}(4,5) \mathrm{P}_{2}-\mathrm{SYT}$ interactions and is remodelled during osmotic stresses.

7. Platre et al., 2018: Proposes the concept of an "electrostatic membrane territory" in plants, which corresponds to post-Golgi membranes.

8. Steffano et al., 2018: Identifies that plant VAP27 proteins directly interact with anionic phospholipids and regulate endocytic trafficking.

9. Simon et al., 2016: Establishes the importance of membrane electrostatics and PI4P in defining the plant PM identity.

10. Ghosh et al., 2015: Proposes a model for Sec14-nodulin function as PI-kinase helper protein mediating $\mathrm{PI}(4,5) \mathrm{P}_{2}$ polar localization. 
- Sidebar (50 words minimum, 200 words maximum) briefly discussing a fascinating adjacent topic. Please give the sidebar a title and insert it below the Literature Cited section, but be sure to call out the sidebar in text; it will be typeset near the section containing the callout. The sidebar cannot contain figures or tables.

Lipid molecular species and lipid packing defects (7): Anionic lipids are defined by their head group. However, they are also constituted of two fatty acid chains that can vary in length and unsaturation degree. These parameters influence membranes physical properties, notably their fluidity and curvature, and thus, the recruitment and dynamics of membrane associated proteins. In addition to membrane charge and curvature, another physical feature of the membrane is of particular interest for protein targeting and is directly influenced by fatty acid composition. Indeed, the density of lipids in the membrane is not homogeneous. The regions with low density are called lipid-packing defects. Membrane curvature and conical shape lipids (which can be linked to the degree of unsaturation) can induce packing defects. In this region, the hydrophobic tails of the lipids are transiently exposed. Membrane associated proteins can recognize and target specifically membrane with packing defects by inserting hydrophobic residues into the membrane. In animal cells, high packing defects are found in ER and cis-Golgi membrane while TGN and PM present low level of packing defects except in highly curved-membranes. The relevance of lipid packing defects for protein recruitment in plants is largely unexplored. 
- Terms and Definitions list: provide definitions for as many as 20 of the most important abbreviations or key terms, limited to 20 words maximum; insert below Literature Cited section

Endomembrane system: membranes system connected directly or through vesicular transport including nuclear envelop, ER, Golgi, PM, endosomes/vesicles, lysosomes/tonoplast, but not chloroplasts/mitochondria.

Lipid Binding Domain (LBD): Protein domain that selectively recognize and target membranes via direct interactions with lipid(s) either via specific, non-specific and/or multivalent interactions.

Electrostatic field: Field created by electric charges that exert forces able to attract or repel other charges entering the field.

Electrostatic membrane territory: Within the endomembrane system, corresponds to negatively-charged membranes, whose cytosolic leaflet harbours anionic phospholipids (PostGolgi compartments), as opposed to neutral membranes (ER-derived).

Nanodomain or nanocluster: Small membrane zone below $100 \mathrm{~nm}$, enriched in one or several lipid species or proteins.

Membrane Contact site (MCS): Close (below 30nm) and stable apposition via tethering elements of two membranes allowing inter-organelle communication, without membrane fusion.

Protein sorting: Delivery process of proteins to their appropriate cellular destination, based on information contained in the protein sequence and/or structure.

Polar secretion: Delivery of proteins or material steered in one or several specific directions within the cell.

Cell plate: Organelle formed by delivery of cell wall and membranes by Golgi-derived vesicles at the plane of cell division in plants.

trans-Golgi Network/Early endosome (TGN/EE): Golgi-derived but Golgi-independent compartment involved in secretion, which also serves as early endosome in plants and involved in endocytic trafficking.

Secretory vesicles (SV): specific subdomain of the TGN/EE made of uncoated vesicles involved in secretion.

Multivesicular bodies (MVB): late endosome characterized by their spherical morphology, and containing intraluminal vesicles destined for vacuolar degradation.

Plasmodesmata (PD): pores that connects plant cells by passing through their cell wall thereby allowing cytoplasmic, PM and ER continuity from cell-to-cell.

Phagophore: a double membrane that encloses and isolates cytoplasmic components during autophagy.

Coincidence binding: Recruitment of a protein at a specific membrane by interaction with 
1234 several membrane landmarks, which together provides high targeting specify.

1235 Lipid transfer protein (LTP): Proteins responsible for shuttling lipids between different 1236 membranes via non-vesicular trafficking.

1237 Flippase: membrane transporters which translocates phospholipids from the luminal to the 1238 cytosolic membrane leaflet, thereby maintaining lipid asymmetry across the bilayer.

1239 Clathrin-coated vesicle (CCV): vesicles decorated by a membrane-deforming Clathrin coat, 1240 involved in many vesicular-mediated trafficking pathway within the cell.

1241 Clathrin-coated pit (CCP): clathrin-coated vesicles at the PM, corresponding to the initial 1242 step of membrane deformation during endocytosis.

1243 Tethering complex: protein complex which brings together two membranes, either 1244 transiently before fusion or in a more sustained manner at contact sites. 


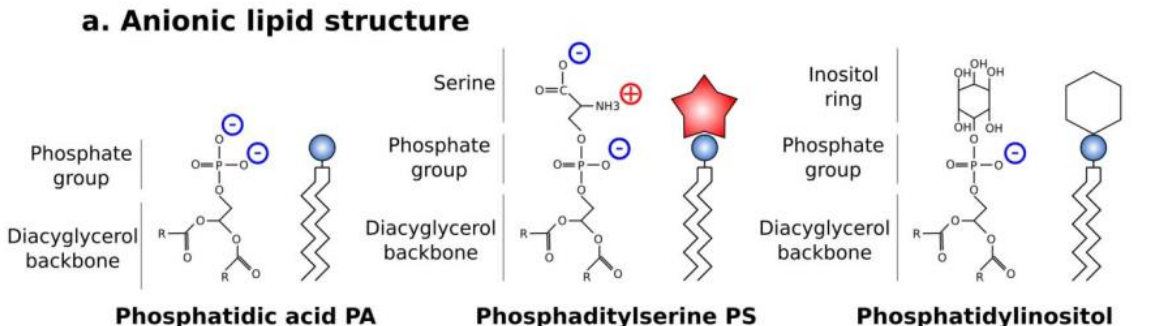

Phosphatidic acid PA

Phosphatidylinositol

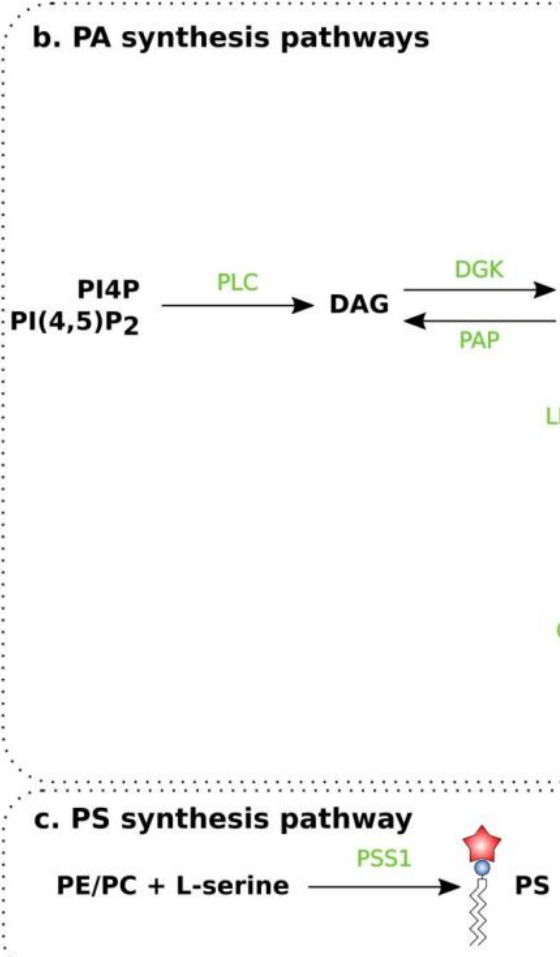

d. PI and PIP synthesis pathways

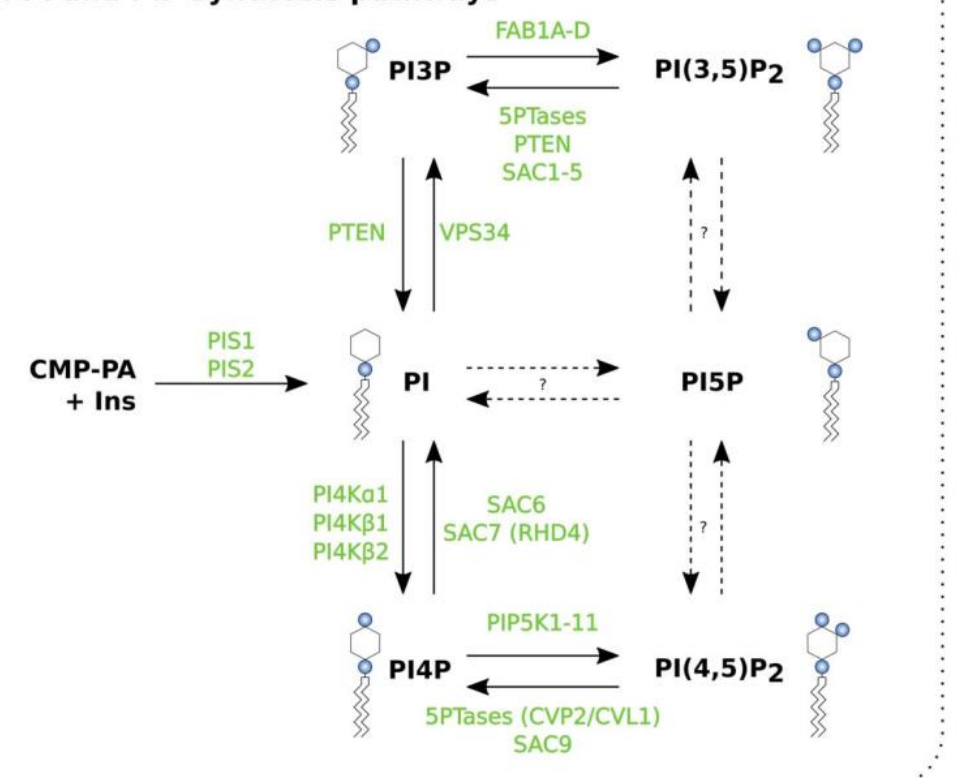

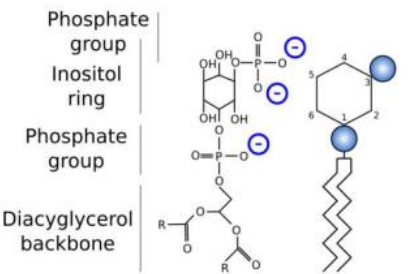

Phosphatidylinositolphosphate or phosphoinositide PIP Ex: PI3P

Figure 1: Anionic lipids.

(a) Skeletal formula and schematic representation of anionic lipids: phosphatidic acid (PA), 
are composed of a diacyglycerol backbone attached to a phosphate group. PS and PI display a serine (star) and an inositol ring (hexagon), respectively, linked to the glycerol by a phosphodiester linkage. PI can be phosphorylated in position 3, 4 and 5 to form 7 different PIPs: Phosphatidylinositol 3-phosphate (PtdIns3P or PI(3)P), Phosphatidylinositol 4phosphate (PtdIns4P or PI(4)P), Phosphatidylinositol 5-phosphate (PtdIns5P or PI(5)P), Phosphatidylinositol 3,4-bisphosphate (PtdIns(3,4)P2 or PI(3,4)P2), Phosphatidylinositol 3,5bisphosphate $(\operatorname{PtdIns}(3,5) \mathrm{P} 2$ or $\mathrm{PI}(3,5) \mathrm{P} 2)$, Phosphatidylinositol 4,5-bisphosphate (PtdIns(4,5)P2, PI(4,5)P2 or PIP2), Phosphatidylinositol 3,4,5-trisphosphate (PtdIns(3,4,5)P3 or PI $\left.(3,4,5) \mathrm{P}_{3}\right)$. Pathways for PA (b), PS (c), PI and PIP (d) production and degradation. PC, phosphatidylcholine; PE, phosphatidylethanolamine; DAG, diacylglycerol; DGPP, Diacylglycerol pyrophosphate; Gly3P, Glycerol 3-phosphate; PLC, Phospholipase C; PLD, Phospholipase D; DGK, Diacylglycerol kinase; PAP, Phosphatidate phosphatase; PAK, PA Kinase; LPP, lipid-phosphate phosphatase; LPAAT, lyso-phosphatidic acid acyltransferase; GPAT, Glycerol-3-phosphate acyltransferase; PSS1, PS Synthase1; CMPPA, Cytidine Monophosphoryl-Phosphatidic Acid; Ins, Inositol; SAC, SUPPRESSOR OF ACTIN; PTEN, PHOPHATASE AND TENSIN Homolog; FAB1, FORMATION OF APLOID AND BINUCLEATE CELLS 1; RHD4, ROOT HAIR DEFECTIVE 4; CVP2, COTYLEDON VASCULAR PATTERN 2; CVL1, CVP2-LIKE1; PI4K, PI 4-Kinases. 


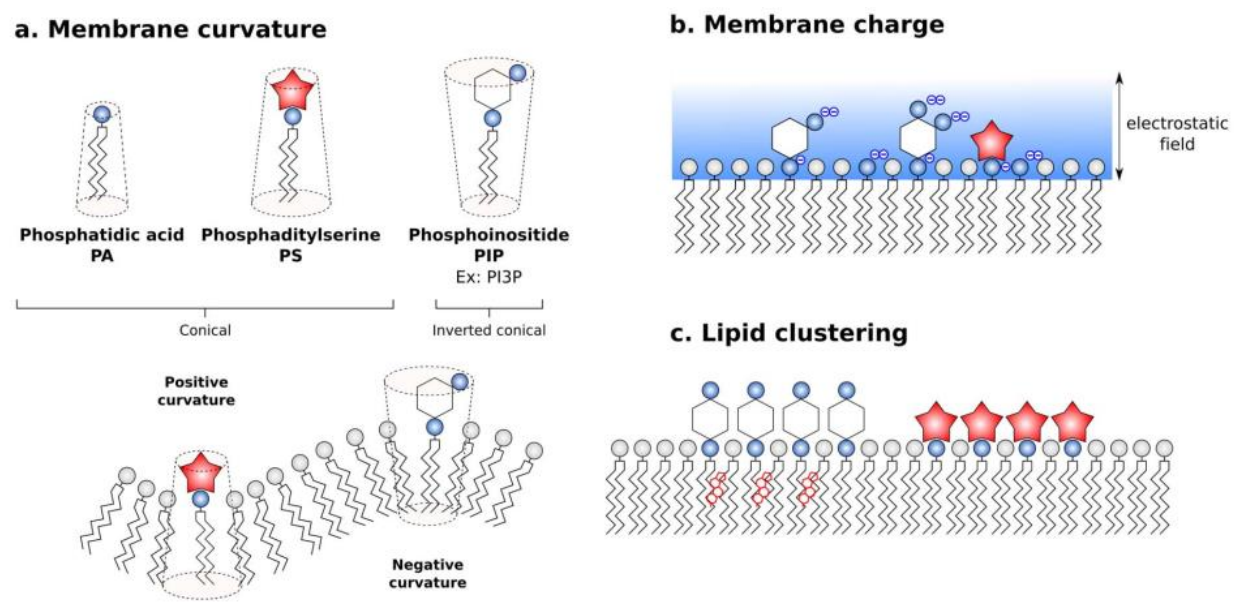

d. Protein membrane targeting by anionic lipid

1273

1274

1275

1276

1277

1278

1279

1280

1281

1282

1283

1284

1285

1286

1287

1288

1289

1290

1291

1292

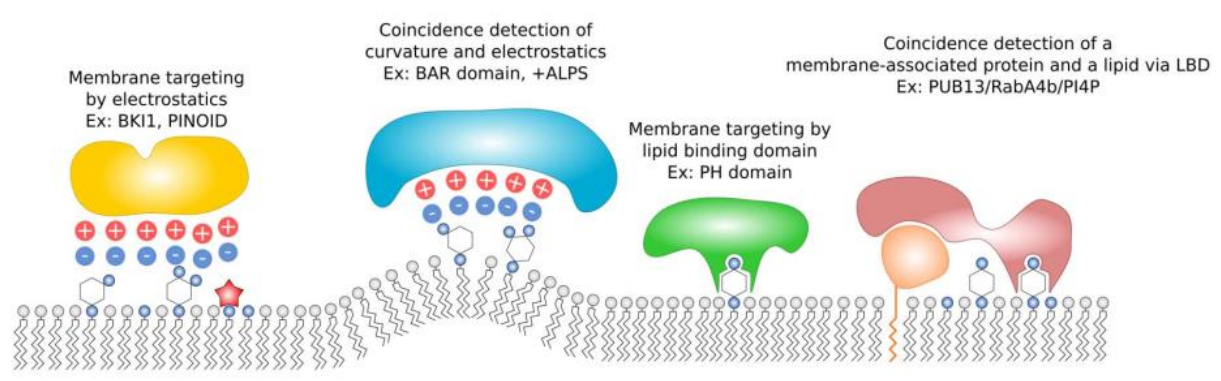

Figure 2: Physicochemical properties of membranes and membrane targeting by anionic lipids.

Anionic lipids can confer several properties to the membranes. (a) Curvature: The geometry of the head group of anionic lipids gives a conic shape to the lipid which influences positively (in the case of PIP) or negatively (in the case of PA and PS) the curvature of the membrane. (b) Electrostatics or membrane surface charge: Accumulation of anionic lipids confers a negative charge to the membrane leading to an electrostatic field. (c) Clustering: Anionic lipids can cluster together to form nanodomains in which one or several lipid species accumulate. (d) These properties can directly participate in the recruitment of proteins to the membrane trough electrostatic interactions between the negative charges of the lipids and the positive charged amino acids, curvature recognition or lipid-binding domain that targets a specific lipid. In some cases, proteins can be recruited to the membrane by coincidence detection of several membrane features (electrostatics and curvature, or an anionic lipid and a membrane-associated protein for instance). Note that for clarity purpose only the cytoplasmic membrane leaflet was drawn. BKI1, BRI1 KINASE INHIBITOR 1; BAR, Bib-AmphyphisinRsv; +ALPS, Amphipathic Lipid Packing Sensor; PH, Pleckstrin Homology; PUB13, PLANT-U-BOX13; PA, Phosphatidic Acid; PS, Phosphatidylserine; PIP, Phosphoinositide; PI3P, Phosphatidylinositol 3-phosphate; PI4P, Phosphatidylinositol 4-phosphate. 

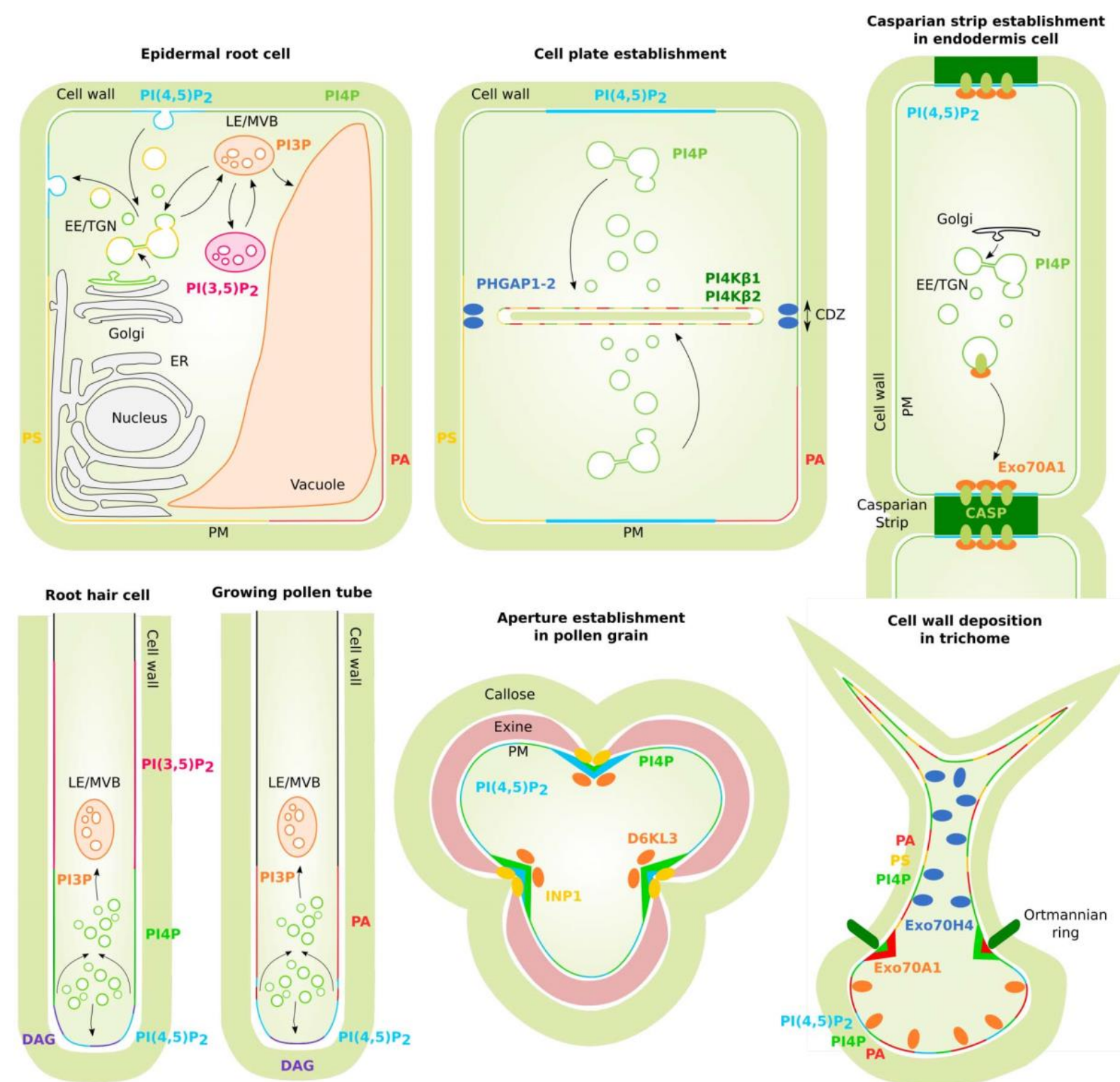

Arbuscular mycorrhizal symbiosis

Colletotrichum infection of root cells
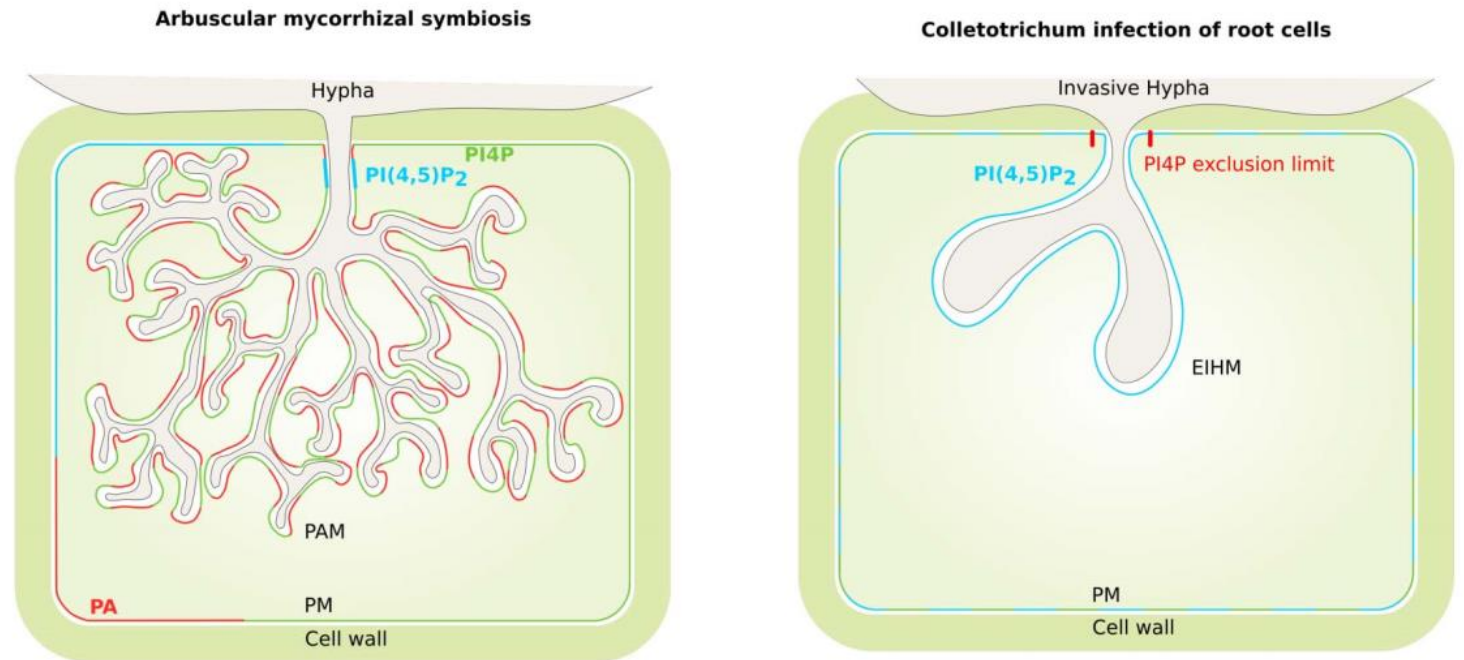

Figure 3: Anionic lipid distribution in plant cells.

The localization of anionic lipids in between the different cellular compartment can change depending of the developmental and physiological context. Here, the distribution of anionic lipids in epidermal root cells is first represented. Anionic lipids can accumulate in specific 
zone of the PM during polarized cell growth (pollen tube, root hair cell for instance), when 1299 interacting with another organism (symbiosis, infection), to determine the position of 1300 developmental structure (aperture of the pollen grains, casparian strip, trichomes). PM, 1301 plasma Membrane; EE/TGN, Early Endosome/Trans-Golgi Network; LE/MVB, Late 1302 Endosome/Multi Vesicular Bodies; ER, Endoplasmic Reticulum; PAM, PeriArbuscular 1303 Membrane; EIHM, Extra Invasive Hyphal Membrane; PA, Phosphatidic Acid; PS, 1304 Phosphatidylserine; PI3P, Phosphatidylinositol 3-phosphate; PI4P, Phosphatidylinositol 41305 phosphate; PI(4,5) $\mathrm{P}_{2}$, Phosphatidylinositol 4,5-bisphosphate; DAG, Diacylglycerol; PI4K,; 1306 PI4P Kinases; PHGAP, Plecktrin Homology GTPase Proteins; D6KL3, D6 PROTEIN 1307 KINASE-LIKE3; INE1, INAPERTURATE POLLEN1; CASP, CASPARIAN STRIP MEMBRANE DOMAIN PROTEIN; Exo70, Exocyst Complex Component 70. 
a. Lipid presentation by SEC14 domain containing protein

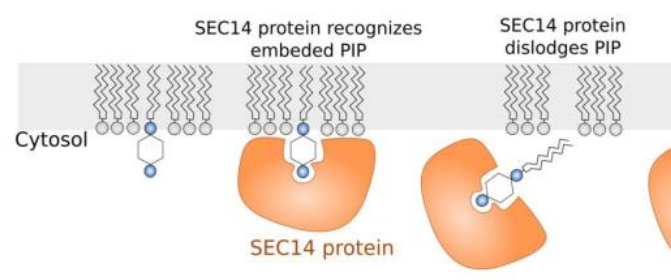

b. PS translocation by flippase
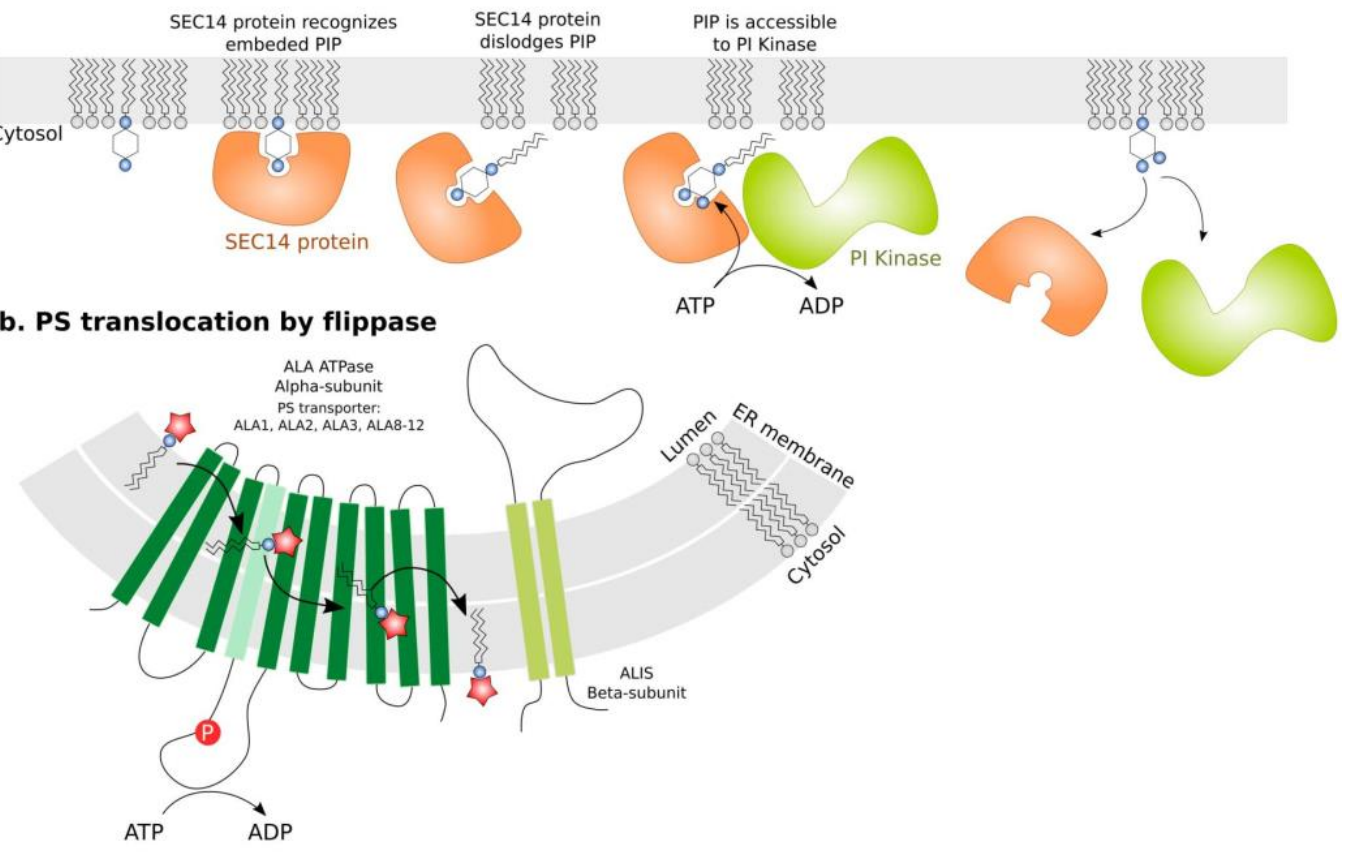

ATP $\quad A D P$

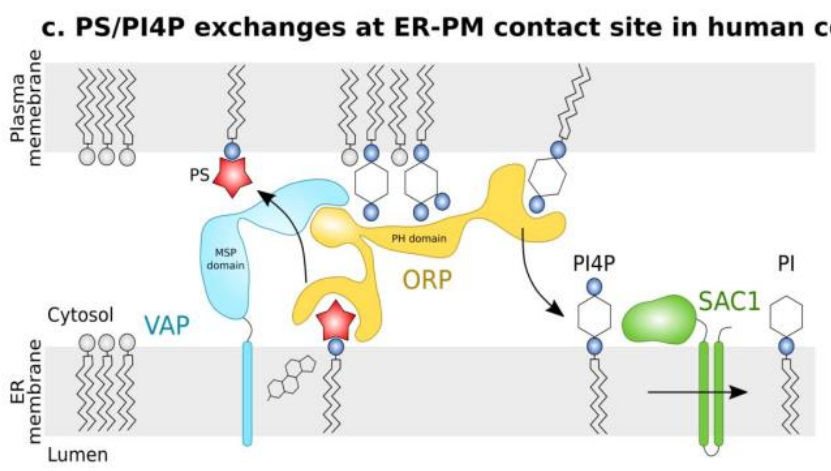

\section{Figure 4: Mechanisms of lipid presentation and transport.}

(a) A mechanism of PIP presentation has been proposed to explain the high processive of PIkinases. In this model, SEC14 protein digs up PI or PIP from the membrane and presents it to the PI-kinase for phosphorylation. (b) The translocation of PS from the luminal leaflet to the cytosolic leaflet of the ER membrane depends on flippase proteins composed of a alpha subunit called ALA which hydrolyses ATP and translocate PS and a $\beta$-subunit called ALIS which specify the localization of the flippase at different membrane compartments and is essential for activity. (c) Lipid exchanges take place at membrane contact sites. In human, ORP5 and 8 tether the ER and the PM by direct interaction with the ER-resident protein VAP and the PM-localized lipid PI4P. ORP5/8 then transfer PS from the ER membrane to the PM via its lipid transfer domain and then counter transport PI4P to the ER. PI4P is then dephosphorylated by SAC1 in the ER membrane maintaining the PI4P gradient between the two membranes. Note that for clarity purpose in panel a and c only the cytoplasmic membrane leaflet was drawn. ER, Endoplasmic Reticulum; EPCS; ER Plasma membrane Contact Site; PS, Phosphatidylserine; PI, Phosphatidylinositol; PIP, Phosphoinositide; SEC14; ALA, aminophospholipid flippase; ALIS, ALA interacting $\beta$-subunit; VAP, VAMP Associated Protein; SAC1, SUPPRESSOR OF ACTIN 1; ATP, Adenosine triphosphate; ADP, Adenosine diphosphate; ORP, OSBP-Related Protein; VAP, vesicle-associated membrane protein-associated proteins. 


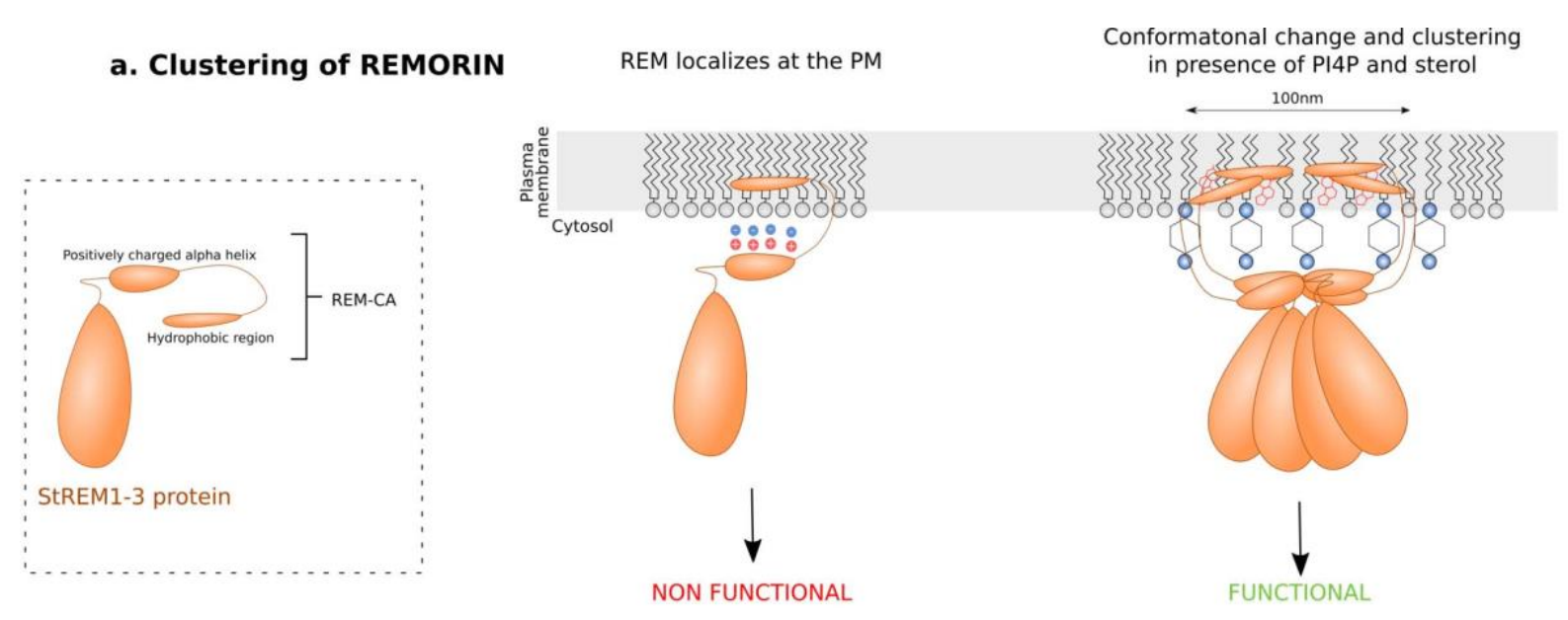

b. Clustering of ROP6

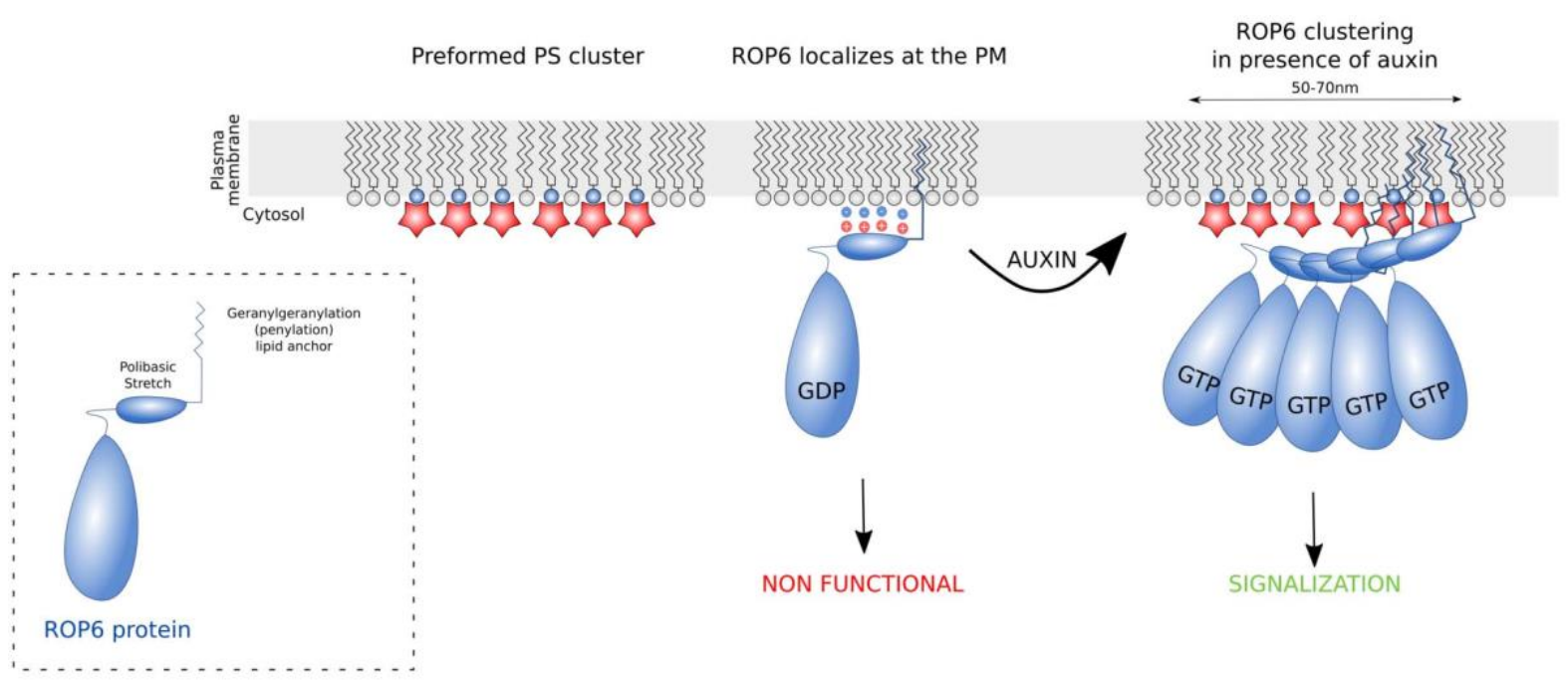

\section{Figure 5: Protein/lipid clustering into nanodomains}

Lipids can accumulate in small regions of the membrane to form nanoclusters in which proteins are constrained. (a) StREM1.3 localizes at the PM through electrostatic interactions thanks to a positively charged alpha helix and hydrophobic interactions via a hydrophobic region that is embedded in the membrane. In presence of PI4P and cholesterol, StREM1.3 undergoes a conformational change that leads to its clustering. The distribution of StREM1.3 in cluster appears necessary to fulfill its function. (b) ROP6 localizes at the PM thanks to a polybasic patch and a lipid anchor. Upon auxin signaling, GTP-bound ROP6 relocalizes in pre-existing PS clusters. The clustering of ROP6 into PS nanodomains is necessary for ROP6 signaling. PM, Plasma Membrane; REM, REMORIN; ROP6, Rho-of-Plant 6; GDP, Guanosine diphosphate; GTP, Guanosine triphosphate; PS, Phosphatidylserine; PI4P, Phosphatidylinositol 4-phosphate. 


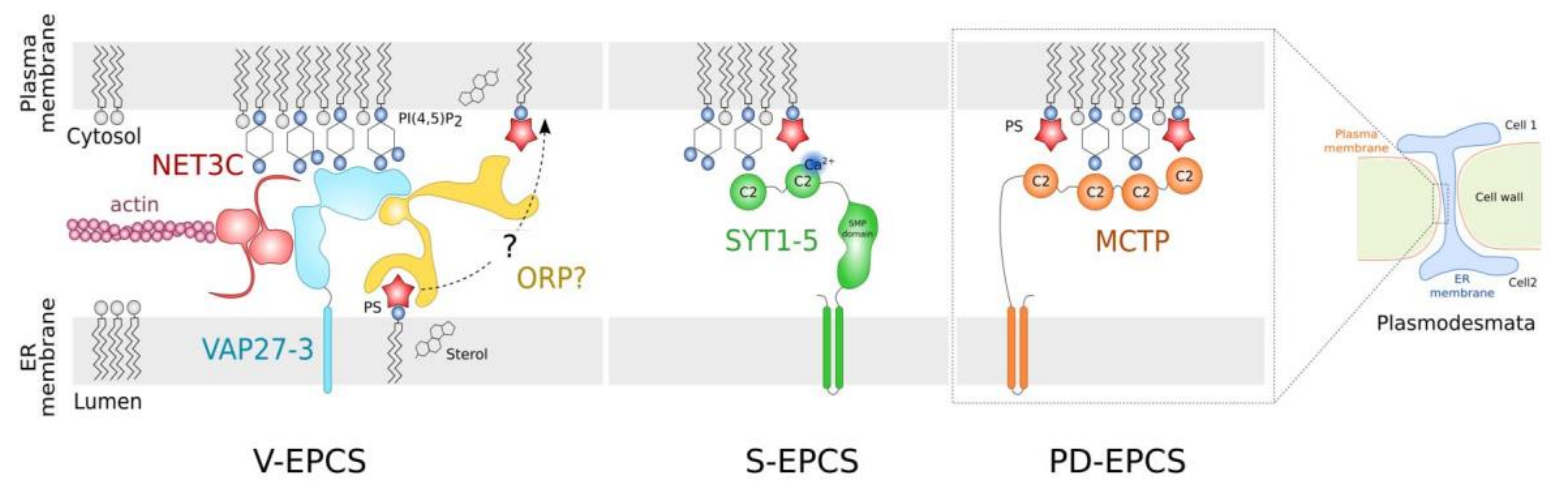

Figure 6: ER-PM Membrane Contact Sites in plant

Schematic representation of the three different classes of EPCS in plant and their relation with anionic lipids. Note that for clarity purpose only the cytoplasmic membrane leaflet was drawn. ER, Endoplasmic Reticulum; EPCS; ER Plasma membrane Contact Site; PD, Plasmodesmata; PS, Phosphatidylserine; PI(4,5)P2, Phosphatidylinositol 4,5-bisphosphate; VAP27, VAMP Associated Protein 27; ORP, OSBP-Related Protein; NET3C, NETWORKED 3C; VAP27, vesicle-associated membrane protein-associated proteins; SYT, SYNAPTOTAGMINE; MCTP, MULTIPLE C2 AND TRANSMEMBRANE PROTEIN, SMP, Synaptotagmin-like mitochondrial-lipid-binding domain.
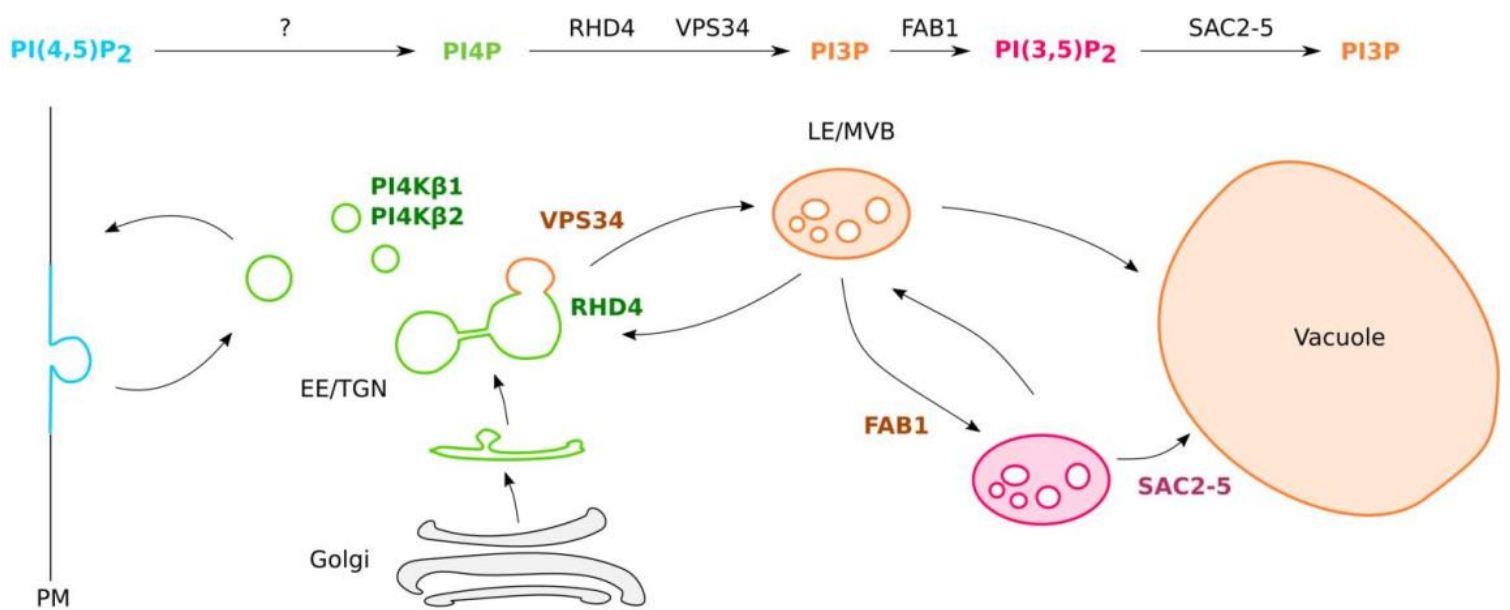

\section{Figure 7: Phosphoinositide cascade during endocytic trafficking to the vacuole}

Kinases and phosphatases create a phosphoinositide cascade along the endocytic route. PM, plasma Membrane; EE/TGN, Early Endosome/Trans-Golgi Network; LE/MVB, Late Endosome/Multi Vesicular Bodies; PI3P, Phosphatidylinositol 3-phosphate; PI4P, Phosphatidylinositol 4-phosphate; $\mathrm{PI}(4,5) \mathrm{P}_{2}$, Phosphatidylinositol 4,5-bisphosphate; $\mathrm{PI}(3,5) \mathrm{P}_{2}$, Phosphatidylinositol 3,5-bisphosphate; PI4K,; PI4P Kinases; RHD4, ROOT HAIR DEFECTIVE 4; FAB1, FORMATION OF APLOID AND BINUCLEATE CELLS 1; SAC, SUPPRESSOR OF ACTIN. 